\title{
Multipower variation for Brownian semistationary processes
}

\author{
OLE E. BARNDORFF-NIELSEN ${ }^{1}$, JOSÉ MANUEL CORCUERA ${ }^{2}$ and \\ MARK PODOLSKIJ ${ }^{3}$ \\ ${ }^{1}$ Department of Mathematical Sciences, University of Aarhus, Ny Munkegade, DK-8000 Aarhus C, \\ Denmark.E-mail: oebn@imf.au.dk \\ ${ }^{2}$ Universitat de Barcelona, Gran Via de les Corts Catalanes 585, 08007 Barcelona, Spain. \\ E-mail: jmcorcuera@ub.edu \\ ${ }^{3}$ Department of Mathematics, ETH Zürich, HG G32.2, 8092 Zürich, Switzerland. \\ E-mail: mark.podolskij@math.ethz.ch
}

In this paper we study the asymptotic behaviour of power and multipower variations of processes $Y$ :

$$
Y_{t}=\int_{-\infty}^{t} g(t-s) \sigma_{s} W(\mathrm{~d} s)+Z_{t}
$$

where $g:(0, \infty) \rightarrow \mathbb{R}$ is deterministic, $\sigma>0$ is a random process, $W$ is the stochastic Wiener measure and $Z$ is a stochastic process in the nature of a drift term. Processes of this type serve, in particular, to model data of velocity increments of a fluid in a turbulence regime with spot intermittency $\sigma$. The purpose of this paper is to determine the probabilistic limit behaviour of the (multi)power variations of $Y$ as a basis for studying properties of the intermittency process $\sigma$. Notably the processes $Y$ are in general not of the semimartingale kind and the established theory of multipower variation for semimartingales does not suffice for deriving the limit properties. As a key tool for the results, a general central limit theorem for triangular Gaussian schemes is formulated and proved. Examples and an application to the realised variance ratio are given.

Keywords: central limit theorem; Gaussian processes; intermittency; non-semimartingales; turbulence; volatility; Wiener chaos

\section{Introduction}

The motivation for the development of the results reported in this paper has been the need to construct tools for studying the probabilistic limit behaviour of (realised) quadratic variation and other multipower variations in relation to the class of Brownian semistationary $(\mathcal{B S S})$ processes. This class, which was introduced in [12], consists of the processes $Y=\left\{Y_{t}\right\}_{t \in \mathbb{R}}$ that are defined by

$$
Y_{t}=\mu+\int_{-\infty}^{t} g(t-s) \sigma_{s} W(\mathrm{~d} s)+\int_{-\infty}^{t} q(t-s) a_{s} \mathrm{~d} s,
$$

where $\mu$ is a constant; $W$ is a Brownian measure on $\mathbb{R} ; g$ and $q$ are non-negative deterministic functions on $\mathbb{R}$, with $g(t)=q(t)=0$ for $t \leq 0$; and $\sigma$ and $a$ are cadlag processes. When $\sigma$ 
and $a$ are stationary, so is $Y$. Hence the name Brownian semistationary processes. It is interesting to note that the fractional Ornstein-Uhlenbeck process is, in fact, also representable in the form (1.1). The same is true of a wide class of stable pseudo-moving average processes; see Corollary 4.3 in [2].

The $\mathcal{B S S}$ processes form the natural analogue, for stationarity related processes, to the class $\mathcal{B S M}$ of Brownian semimartingales

$$
Y_{t}=\mu+\int_{0}^{t} \sigma_{s} \mathrm{~d} W_{s}+\int_{0}^{t} a_{s} \mathrm{~d} s .
$$

In the context of stochastic modelling in finance and in turbulence, the process $\sigma$ embodies the volatility or intermittency of the dynamics, whether the framework is that of $\mathcal{B S} M$ or $\mathcal{B S S}$. For detailed discussion of $\mathcal{B S S}$ and the more general concept of tempo-spatial ambit processes see [7-12]. Such processes are, in particular, able to reproduce key stylized features of turbulent data.

A main difference between $\mathcal{B S} \mathcal{M}$ and $\mathcal{B S} S$ is that, in general, models of the $\mathcal{B S S}$ form are not semimartingales (for a discussion of this, see Section 3 of [12]). In consequence, various important techniques developed for semimartingales, such as the calculation of quadratic variation by Itô calculus and those of multipower variation, do not apply or suffice in $\mathcal{B S S}$ settings. The present paper addresses some of the issues that this raises.

The theory of multipower variation was primarily developed as a basis for inference on $\sigma$ under $\mathcal{B S} \mathcal{M}$ models and, more generally, Itô semimartingales, with particular focus on inference about the integrated squared volatility $\sigma^{2+}$ given by

$$
\sigma_{t}^{2+}=\int_{0}^{t} \sigma_{s}^{2} \mathrm{~d} s .
$$

This quantity is likewise a focal point for the results discussed in the following.

Section 2 introduces common notation for multipower variation and recalls some basic properties of such quantities. A law of large numbers and a central limit theorem for multipower variation of triangular arrays of Gaussian random variables are derived in Section 3, and these limit results are drawn upon in Section 4 to establish probability and central limit theorems for multipower variation for $\mathcal{B S S}$ processes, with most of the proofs postponed to Section 8. Section 5 presents several examples and Section 6 discusses an application concerning the limit behaviour of the realised variation ratio, that is, the ratio of realised bipower variation to realised quadratic variation. Section 7 concludes and indicates some possible directions for further related work.

\section{Multipower variation}

The concept of (realised) multipower variation was originally introduced in [13] in the context of semimartingales, and the mathematical theory has been studied in a number of papers $[6,17,24$, 27] while various applications are the main subjects in [14-16,25,33]. Multipower variation turns out to be useful for analysing properties of parts of a process that are not directly observable. In 
this section we present the definition of realised multipower variation and recall its asymptotic properties for some classes of processes.

Let us consider a continuous-time process $X$, defined on some filtered probability space $\left(\Omega, \mathcal{F},\left(\mathcal{F}_{t}\right)_{t \geq 0}, P\right)$, that is observed at equidistant time points $t_{i}=i / n, i=0, \ldots,[n t]$. A realised multipower variation of the process $X$ is an object of the type

$$
\sum_{i=1}^{[n t]-k+1} \prod_{j=1}^{k}\left|\Delta_{i+j-1}^{n} X\right|^{p_{j}}, \quad \Delta_{i}^{n} X=X_{i / n}-X_{(i-1) / n}, \quad p_{1}, \ldots, p_{k} \geq 0
$$

for some fixed number $k \geq 1$. We now present an overview of the asymptotic theory for quantities of the form (2.1) for various types of processes $X$.

We start with the $\mathcal{B S} \mathcal{M}$ case

$$
X_{t}=X_{0}+\int_{0}^{t} a_{s} \mathrm{~d} s+\int_{0}^{t} \sigma_{s} \mathrm{~d} W_{s}
$$

where $W$ is a Brownian motion, $a$ is a locally bounded and predictable drift process and $\sigma$ is an adapted and cadlag volatility process. As was established in [6], the convergence in probability

$$
n^{p_{+} / 2-1} \sum_{i=1}^{[n t]-k+1} \prod_{j=1}^{k}\left|\Delta_{i+j-1}^{n} X\right|^{p_{j}} \stackrel{\text { ucp }}{\longrightarrow} \mu_{p_{1}} \cdots \mu_{p_{k}} \int_{0}^{t}\left|\sigma_{s}\right|^{p_{+}} \mathrm{d} s
$$

holds, where $p_{+}=\sum_{p=1}^{k} p_{j}$ and $\mu_{p}=E\left[|u|^{p}\right], u \sim N(0,1)$ and we write $Z^{n} \stackrel{\text { ucp }}{\longrightarrow} Z$ when $\sup _{t \in[0, T]}\left|Z_{t}^{n}-Z_{t}\right| \stackrel{P}{\longrightarrow} 0$ for any $T>0$. Under a further condition on the volatility process, one obtains the associated stable central limit theorem:

$$
\begin{aligned}
& \sqrt{n}\left(n^{p_{+} / 2-1} \sum_{i=1}^{[n t]-k+1} \prod_{j=1}^{k}\left|\Delta_{i+j-1}^{n} X\right|^{p_{j}}-\mu_{p_{1}} \cdots \mu_{p_{k}} \int_{0}^{t}\left|\sigma_{s}\right|^{p_{+}} \mathrm{d} s\right) \\
& \stackrel{\mathrm{st}}{\longrightarrow} \sqrt{A} \int_{0}^{t}\left|\sigma_{s}\right|^{p_{+}} \mathrm{d} B_{s},
\end{aligned}
$$

where $B$ is another Brownian motion, defined on an extension of the probability space $\left(\Omega, \mathcal{F},\left(\mathcal{F}_{t}\right)_{t \geq 0}, P\right)$ and independent of $\mathcal{F}$, and the constant $A$ is given by

$$
A=\prod_{l=1}^{k} \mu_{2 p_{l}}-(2 k-1) \prod_{l=1}^{k} \mu_{p_{l}}^{2}+2 \sum_{m=1}^{k-1} \prod_{l=1}^{m} \mu_{p_{l}} \prod_{l=k-m+1}^{k} \mu_{p_{l}} \prod_{l=1}^{k-m} \mu_{p_{l}+p_{l+m}} .
$$

Recall that the stable convergence of processes is defined as follows. A sequence of processes $Z^{n}$ converges stably in law towards the process $Z$ (written $Z^{n} \stackrel{\text { st }}{\longrightarrow} Z$ ), that is, defined on the extension of the original probability space $\left(\Omega, \mathcal{F},\left(\mathcal{F}_{t}\right)_{t \geq 0}, P\right)$, if and only if for any bounded 
and continuous real-valued functional $f$ and any $\mathcal{F}$-measurable random variable $V$ it holds that

$$
\lim _{n \rightarrow \infty} E\left[f\left(Z^{n}\right) V\right]=E[f(Z) V],
$$

we use the notation $Z^{n} \stackrel{\text { st }}{\longrightarrow} Z$.

A crucial property of the realised multipower variation is its robustness to jumps when $\max _{i}\left(p_{i}\right)<2[17,26]$. Assume for a moment that $X$ is a general Itô semimartingale with continuous part $X^{c}$ satisfying (2.2). Then, by (2.3) and the robustness property, we obtain the convergence

$$
\frac{\mu_{1}^{-2} \sum_{i=1}^{[n t]-1}\left|\Delta_{i}^{n} X\right|\left|\Delta_{i+1}^{n} X\right|}{\sum_{i=1}^{[n t]}\left|\Delta_{i}^{n} X\right|^{2}} \stackrel{P}{\longrightarrow} \frac{\left[X^{c}\right]}{[X]},
$$

where $[X]$ denotes the quadratic variation of the semimartingale $X$ and the limit is less than or equal to 1 . The latter result, together with the stable convergence in (2.4), can be used to construct a formal test for jumps (see [13]). On the other hand, we know that if the limit of the left-hand side is greater than 1 (which is the case for some typical turbulence data), the process $X$ cannot be an Itô semimartingale.

In another direction, a study [5] was made of the asymptotic behaviour of bipower variation for processes of the type

$$
X_{t}=X_{0}+\int_{0}^{t} \sigma_{s} \mathrm{~d} G_{s}, \quad t \geq 0,
$$

where $G$ is a continuous Gaussian process with centered and stationary increments (the latter integral is defined as a Riemann-Stieltjes integral). The process defined in (2.5) is, in general, also not a semimartingale, and the theory in [6] does not apply. In particular, a different normalisation is required. Define the (normalised) multipower variation by

$$
V\left(X, p_{1}, \ldots, p_{k}\right)_{t}^{n}=\frac{1}{n \tau_{n}^{p_{+}}} \sum_{i=1}^{[n t]-k+1} \prod_{j=1}^{k}\left|\Delta_{i+j-1}^{n} X\right|^{p_{j}}, \quad p_{1}, \ldots, p_{k} \geq 0,
$$

where $\tau_{n}>0$ is given by

$$
\tau_{n}^{2}=\bar{R}[(1 / n)]
$$

with

$$
\bar{R}(t)=E\left[\left(G_{s+t}-G_{S}\right)^{2}\right] .
$$

Under some assumptions on $\bar{R}$ and the volatility process $\sigma$ it was shown that

$$
V\left(X, p_{1}, \ldots, p_{k}\right)_{t}^{n} \stackrel{\text { ucp }}{\longrightarrow} \rho_{p_{1}, \ldots, p_{k}} \int_{0}^{t}\left|\sigma_{s}\right|^{p_{+}} \mathrm{d} s
$$

for a certain constant $\rho_{p_{1}, \ldots, p_{k}}$ that depends on the behaviour of $\bar{R}$ near 0 . Furthermore, an associated (stable) central limit theorem, of a form similar to (2.4), was derived. Note, however, that 
in general there are essential differences between the characters of $\mathcal{B S S}$ processes and processes of type (2.5). In the latter case, the process $\sigma$ has only a local influence in the value of $X$ whereas, in the $\mathcal{B S S}$ case, the process is also affected by the past of $\sigma$.

\section{Multipower variation of Gaussian triangular arrays}

In this section we derive some asymptotic results for functionals of arrays of stationary Gaussian sequences. We consider a triangular array $\left(X_{i, n}\right)_{n \geq 1,1 \leq i \leq[n t]}(t>0)$ of row-wise stationary Gaussian variables with mean 0 and variance 1 . Let

$$
r_{n}(j)=\operatorname{cor}\left(X_{1, n}, X_{1+j, n}\right), \quad j \geq 0,
$$

be the correlation function of $\left(X_{i, n}\right)_{1 \leq i \leq[n t]}$. Assume that the array $\left(X_{i, n}\right)_{n \geq 1,1 \leq i \leq[n t]}$ is "nondegenerate", that is, the covariance matrix of $\left(X_{i, n}, \ldots, X_{i+k, n}\right)$ is invertible for any $k \geq 1$ and $n \geq 1$ (otherwise the results below do not hold).

Now, define the multipower variation associated with the sequence $\left(X_{i, n}\right)_{n \geq 1,1 \leq i \leq[n t]}$ :

$$
V\left(p_{1}, \ldots, p_{k}\right)_{t}^{n}=\frac{1}{n} \sum_{i=1}^{[n t]-k+1} \prod_{j=1}^{k}\left|X_{i+j-1, n}\right|^{p_{j}}, \quad p_{1}, \ldots, p_{k} \geq 0 .
$$

Our first result is the weak law of large numbers.

Theorem 1. Assume that there exists a sequence $\mathrm{r}(j)$ with

$$
r_{n}^{2}(j) \leq \mathrm{r}(j), \quad \frac{1}{n} \sum_{j=1}^{n-1} \mathrm{r}(j) \rightarrow 0
$$

as $n \rightarrow \infty$. Then it holds that

$$
V\left(p_{1}, \ldots, p_{k}\right)_{t}^{n}-\rho_{p_{1}, \ldots, p_{k}}^{(n)} t \stackrel{\text { ucp }}{\longrightarrow} 0,
$$

where

$$
\rho_{p_{1}, \ldots, p_{k}}^{(n)}=E\left[\left|X_{1, n}\right|^{p_{1}} \cdots\left|X_{k, n}\right|^{p_{k}}\right]
$$

Proof. See Section 8.

Before we present the associated central limit theorem, we need to introduce another Gaussian process. Suppose that $r_{n}(j) \rightarrow \rho(j), j=1, \ldots, k-1$, for some numbers $\rho(j)$. Let $\left(Q_{i}\right)_{i \geq 1}$ be a non-degenerate, stationary, centered (discrete-time) Gaussian process with variance 1 and correlation function

$$
\rho(j)=\operatorname{cor}\left(Q_{1}, Q_{1+j}\right), \quad j \geq 1
$$


Define

$$
V_{Q}\left(p_{1}, \ldots, p_{k}\right)_{t}^{n}=\frac{1}{n} \sum_{i=1}^{[n t]-k+1} \prod_{j=1}^{k}\left|Q_{i+j-1}\right|^{p_{j}}
$$

and let $\rho_{p_{1}, \ldots, p_{k}}=E\left[\left|Q_{1}\right|^{p_{1}} \ldots\left|Q_{k}\right|^{p_{k}}\right]$. Then $\rho_{p_{1}, \ldots, p_{k}}^{(n)} \rightarrow \rho_{p_{1}, \ldots, p_{k}}$ and in this case we obtain the uniform convergence on compacts in probability (or ucp convergence):

$$
V\left(p_{1}, \ldots, p_{k}\right)_{t}^{n} \stackrel{\text { ucp }}{\longrightarrow} \rho_{p_{1}, \ldots, p_{k}} t .
$$

Now we have the following central limit theorem for the family $\left(V\left(p_{1}^{j}, \ldots, p_{k}^{j}\right)_{t}^{n}\right)_{1 \leq j \leq d}$.

Theorem 2. Assume that

$$
r_{n}(j) \rightarrow \rho(j), \quad j \geq 0,
$$

and that, for any $j, n \geq 1$, there exists a sequence $\mathrm{r}(j)$ with

$$
r_{n}^{2}(j) \leq \mathrm{r}(j), \quad \sum_{j=1}^{\infty} \mathrm{r}(j)<\infty .
$$

Then we have

$$
\sqrt{n}\left(V\left(p_{1}^{j}, \ldots, p_{k}^{j}\right)_{t}^{n}-\rho_{p_{1}^{j}, \ldots, p_{k}^{j}}^{(n)} t\right)_{1 \leq j \leq d} \stackrel{\mathcal{L}}{\rightarrow} \beta^{1 / 2} B_{t},
$$

where $B$ is a d-dimensional Brownian, $\beta$ is a $d \times d$-dimensional matrix given by

$$
\beta_{i j}=\lim _{n \rightarrow \infty} n \operatorname{cov}\left(V_{Q}\left(p_{1}^{i}, \ldots, p_{k}^{i}\right)_{1}^{n}, V_{Q}\left(p_{1}^{j}, \ldots, p_{k}^{j}\right)_{1}^{n}\right), \quad 1 \leq i, j \leq d,
$$

and the weak convergence holds in the space $\mathcal{D}\left([0, T]^{d}\right)$ equipped with the uniform topology.

Proof. See Section 8 .

Remark 1. Similar asymptotic results can be obtained for general quantities of the form

$$
\frac{1}{n} \sum_{i=1}^{[n t]-k+1} H\left(X_{i, n}, \ldots, X_{i+k-1, n}\right)
$$

for some function $H: \mathbb{R}^{k} \rightarrow \mathbb{R}$. Let $m$ denote the Hermite index of $H$ (notice that the Hermite index of the power function used in (3.2) is 2). Replace condition (3.3) by

$$
\left|r_{n}^{m}(j)\right| \leq \mathrm{r}(j), \quad \frac{1}{n} \sum_{j=1}^{n-1} \mathrm{r}(j) \rightarrow 0
$$


and (3.9) by

$$
\left|r_{n}^{m}(j)\right| \leq \mathrm{r}(j), \quad \sum_{j=1}^{\infty} \mathrm{r}(j)<\infty .
$$

Then Theorems 1 and 2 hold true for the functional (3.12) provided that $E H^{2}\left(N_{k}(0, \Sigma)\right)<\infty$ for any invertible $\Sigma \in \mathbb{R}^{k \times k}$. We omit the details.

Remark 2. Ho and Sun [23] have shown a non-functional version of Theorem 2 for statistics of the type (3.12) when the correlation function $r_{n}$ does not depend on $n$. To the best of our knowledge, Theorem 2 is the first central limit theorem for (general) multipower variation of a row-wise stationary Gaussian process.

\section{Multipower variation for $\mathcal{B S S}$ processes}

Armed with the general theorems proved in Section 3, we are now set to establish laws of large numbers and central limit results for multipower variations in the framework of the Brownian semistationary processes. The regularity conditions invoked are given in a first subsection, while the next states the theorems, the main parts of the proofs being postponed to Section 8; the third subsection discusses the nature of the rather technical regularity conditions and describes a set of simpler assumptions that are more amenable to checking.

\subsection{Conditions}

We consider a filtered probability space $\left(\Omega, \mathcal{F},\left(\mathcal{F}_{t}\right)_{t \geq 0}, P\right)$, assuming the existence thereon of a $\mathcal{B S S}$ process, without drift term for the time being, that is,

$$
Y_{t}=\int_{-\infty}^{t} g(t-s) \sigma_{s} W(\mathrm{~d} s),
$$

where $W$ is an $\left(\mathcal{F}_{t}\right)$-Brownian measure on $\mathbb{R}, \sigma$ is an $\left(\mathcal{F}_{t}\right)$-adapted and cadlag volatility process and $g: \mathbb{R} \rightarrow \mathbb{R}$ is a deterministic continuous memory function with $g(t)=0$ for $t \leq 0$ and $g \in$ $L^{2}((0, \infty))$. We also require $\int_{-\infty}^{t} g^{2}(t-s) \sigma_{s}^{2} \mathrm{~d} s<\infty$ a.s. to ensure that $Y_{t}<\infty$ a.s. for all $t \geq 0$. By an $\left(\mathcal{F}_{t}\right)$-Brownian measure we understand a Gaussian stochastic measure such that, for any Borelian set $A$ with $E\left[W(A)^{2}\right]<\infty$,

$$
W(A) \sim N(0, m(A)),
$$

where $m$ is the Lebesgue measure, and if $A \subseteq[t,+\infty)$, then $W(A)$ is independent of $\mathcal{F}_{t}$. Note that $\left\{B_{t}:=\int_{a}^{t} W(\mathrm{~d} s), t \geq a\right\}$ is a standard Brownian motion starting in $a$.

The process $Y$ is assumed to be observed at time points $t_{i}=i / n, i=1, \ldots,[n t]$. Now, let $G$ be the stationary Gaussian process defined as

$$
G_{t}=\int_{-\infty}^{t} g(t-s) W(\mathrm{~d} s) .
$$


This is an important auxiliary object in the study of $\mathcal{B S S}$ processes. Note that $G$ belongs to the type of processes occurring in (2.5), and that the autocorrelation function of $G$ is

$$
r(t)=\frac{\int_{0}^{\infty} g(t+u) g(u) \mathrm{d} u}{\int_{0}^{\infty} g^{2}(u) \mathrm{d} u} .
$$

We are interested in the asymptotic behaviour of the functionals

$$
V\left(Y, p_{1}, \ldots, p_{k}\right)_{t}^{n}=\frac{1}{n \tau_{n}^{p_{+}}} \sum_{i=1}^{[n t]-k+1} \prod_{j=1}^{k}\left|\Delta_{i+j-1}^{n} Y\right|^{p_{j}}, \quad p_{1}, \ldots, p_{k} \geq 0
$$

where $\Delta_{i}^{n} Y=Y_{i / n}-Y_{(i-1) / n}$ and $\tau_{n}^{2}=\bar{R}(1 / n)$ with $\bar{R}(t)=E\left[\left|G_{s+t}-G_{s}\right|^{2}\right], t \geq 0$. In the following we assume that the function $g$ is continuously differentiable on $(0, \infty),\left|g^{\prime}\right|$ is nonincreasing on $(b, \infty)$ for some $b>0$ and $g^{\prime} \in L^{2}((\varepsilon, \infty))$ for any $\varepsilon>0$. Moreover, we assume that for any $t>0$,

$$
F_{t}=\int_{1}^{\infty}\left(g^{\prime}(s)\right)^{2} \sigma_{t-s}^{2} \mathrm{~d} s<\infty
$$

almost surely.

Remark 3. Assumption (4.4) ensures that the process $Y$ has the same "smoothness" as the process $G$ (see Lemma 1 in Section 8). It is rather easy to check in practice, because it is implied by the condition $E F_{t}<\infty$ for $t>0$. Furthermore, if $g$ has bounded support, assumption (4.4) is trivially fulfilled since $\sigma$ is cadlag.

Remark 4. Let us note again that the process $Y$ is, in general, not a semimartingale. In particular, this is the case when $g^{\prime} \notin L^{2}((0, \infty))$. For a closer discussion, see [12]. On the other hand, the process $Y$ is not of the form (2.5). Thus, we require new methods to prove the asymptotic results for $V\left(Y, p_{1}, \ldots, p_{k}\right)_{t}^{n}$. Processes of the form (4.1) are used for modelling velocity of turbulent flows; see [8-10]. The function $g$, which is used in such models, behaves often as $x^{\delta}$ near the origin. Hence, when $\delta \in(-1 / 2,1 / 2) \backslash\{0\}, Y$ is neither a differentiable process nor a semimartingale (because $\left.g^{\prime} \notin L^{2}((0, \infty))\right)$. This is the primary case of our interest.

We define the correlation function of the increments of $G$ :

$$
r_{n}(j)=\operatorname{cov}\left(\frac{\Delta_{1}^{n} G}{\tau_{n}}, \frac{\Delta_{1+j}^{n} G}{\tau_{n}}\right)=\frac{\bar{R}((j+1) / n)+\bar{R}((j-1) / n)-2 \bar{R}(j / n)}{2 \tau_{n}^{2}}, \quad j \geq 0 .
$$

Next, we introduce a class of measures that is crucial for our purposes. We define (recall that $g(x):=0$ for $x \leq 0$ )

$$
\pi^{n}(A)=\frac{\int_{A}(g(x-1 / n)-g(x))^{2} \mathrm{~d} x}{\int_{0}^{\infty}(g(x-1 / n)-g(x))^{2} \mathrm{~d} x}, \quad A \in \mathcal{B}(\mathbb{R}) .
$$

Note that $\pi^{n}$ is a probability measure on $\mathbb{R}_{+}$. 
For the weak law of large numbers we require the following assumptions:

(LLN) There exists a sequence $r(j)$ with

$$
r_{n}^{2}(j) \leq \mathrm{r}(j), \quad \frac{1}{n} \sum_{j=1}^{n-1} \mathrm{r}(j) \rightarrow 0 .
$$

Moreover, it holds that

$$
\lim _{n \rightarrow \infty} \pi^{n}((\varepsilon, \infty))=0
$$

for any $\varepsilon>0$.

\section{Remark 5.}

(i) The first condition of (LLN) is adapted from Theorem 1. It guarantees the ucp convergence of $V\left(G, p_{1}, \ldots, p_{k}\right)_{t}^{n}$. The second condition of (LLN) says that the whole mass of the measure $\pi^{n}$ concentrates at 0 . In particular, it is equivalent to the weak convergence

$$
\pi^{n} \rightarrow \delta_{0}
$$

where $\delta_{0}$ is the Dirac measure at 0 .

(ii) Condition (4.6) is absolutely crucial for the limit theorems given in the next subsection. When this condition is violated things become more complicated. In particular, it may lead to a different stochastic limit of $V\left(Y, p_{1}, \ldots, p_{k}\right)_{t}^{n}$ (see the first example in Section 5). Intuitively, this can be explained by the observation that the increments $\Delta_{i}^{n} Y$ contain substantial information about the volatility (far) outside of the interval $\left[\frac{i-1}{n}, \frac{i}{n}\right]$ when condition (4.6) does not hold. Thus, in general, we can not expect the limit described in Theorem 3 below.

Now, we introduce the assumptions for the central limit theorem:

(CLT) Assumption (LLN) holds, and

$$
r_{n}(j) \rightarrow \rho(j), \quad j \geq 0,
$$

where $\rho(j)$ is the correlation function of $\left(Q_{i}\right)_{i \geq 1}$, as introduced in (3.6). Furthermore, there exists a sequence $\mathrm{r}(j)$ such that, for any $j, n \geq 1$,

$$
r_{n}^{2}(j) \leq \mathrm{r}(j), \quad \sum_{j=1}^{\infty} \mathrm{r}(j)<\infty
$$

and for some $\gamma \in(0,1]$ we have

$$
E\left[\left|\sigma_{t}-\sigma_{s}\right|^{A}\right] \leq C|t-s|^{A \gamma}
$$


for any $A>0$. Finally, set $p=\min _{1 \leq i \leq k, 1 \leq j \leq d}\left(p_{i}^{j}\right)$. Assume that $\gamma(p \wedge 1)>\frac{1}{2}$ and that there exists a constant $\lambda<-\frac{1}{p \wedge 1}$ such that for any $\varepsilon_{n}=\mathrm{O}\left(n^{-\kappa}\right), \kappa \in(0,1)$, we have

$$
\pi^{n}\left(\left(\varepsilon_{n}, \infty\right)\right)=\mathrm{O}\left(n^{\lambda(1-\kappa)}\right)
$$

\subsection{Limit theorems}

In this section we present the limit laws of multipower variations of $\mathcal{B S} \mathcal{S}$ processes, in part widening the scope slightly to allow more general drift terms. Recall that the (realised) multipower variation of a process $Y$ of the form (4.1) is defined as

$$
V\left(Y, p_{1}, \ldots, p_{k}\right)_{t}^{n}=\frac{1}{n \tau_{n}^{p_{+}}} \sum_{i=1}^{[n t]-k+1} \prod_{j=1}^{k}\left|\Delta_{i+j-1}^{n} Y\right|^{p_{j}}, \quad p_{1}, \ldots, p_{k} \geq 0,
$$

where $\tau_{n}^{2}=\bar{R}\left(\frac{1}{n}\right)$ and $p_{+}=\sum_{j=1}^{k} p_{j}$. Our first result is the following probability limit theorem.

Theorem 3. Consider a process $Z=Z_{1}+Z_{2}$, where $Z_{2}=Y$ is given by (4.1). Assume that the condition (LLN) holds and that

$$
\frac{1}{n \tau_{n}^{p_{+}}} \sum_{i=1}^{[n t]-k+1} \prod_{j=1}^{k}\left|\Delta_{i+j-1}^{n} Z_{\iota_{j}}\right|^{p_{j}} \stackrel{P}{\longrightarrow} 0
$$

where $\iota_{1}, \ldots, \iota_{k} \in\{1,2\}$, for any $t>0$ and any $\left(\iota_{1}, \ldots, \iota_{k}\right) \neq(2, \ldots, 2)$. Define

$$
\rho_{p_{1}, \ldots, p_{k}}^{(n)}=E\left[\left|\frac{\Delta_{1}^{n} G}{\tau_{n}}\right|^{p_{1}} \ldots\left|\frac{\Delta_{k}^{n} G}{\tau_{n}}\right|^{p_{k}}\right] .
$$

Then we have

$$
V\left(Z, p_{1}, \ldots, p_{k}\right)_{t}^{n}-\rho_{p_{1}, \ldots, p_{k}}^{(n)} \int_{0}^{t}\left|\sigma_{s}\right|^{p_{+}} \mathrm{d} s \stackrel{\text { ucp }}{\longrightarrow} 0 .
$$

Proof. See Section 8 .

Remark 6. The multipower variation is robust to drift processes $Z_{1}$ that are smoother than the process $Y$. Assume, for instance, that the process $Z_{1}$ satisfies

$$
E\left[\left|Z_{1}(t)-Z_{1}(s)\right|^{p}\right]=\mathrm{o}\left(\bar{R}^{p / 2}(|t-s|)\right)
$$

for every $p>0$. In this case, condition (4.10) is obviously satisfied.

Next, we demonstrate a joint central limit theorem for a family $\left(V\left(Z, p_{1}^{j}, \ldots, p_{k}^{j}\right)_{t}^{n}\right)_{1 \leq j \leq d}$ of multipower variations. 
Theorem 4. Consider a process $Z=Z_{1}+Z_{2}$, where $Z_{2}=Y$ is given by (4.1). Assume that the condition (CLT) holds and that

$$
\frac{1}{\sqrt{n} \tau_{n}^{p_{+}^{j}}} \sum_{i=1}^{[n t]-k+1} \prod_{l=1}^{k}\left|\Delta_{i+l-1}^{n} Z_{i_{l}}\right|^{p_{l}^{j}} \stackrel{P}{\longrightarrow} 0
$$

where $\iota_{1}, \ldots, \iota_{k} \in\{1,2\}$, for any $t>0$ and any $\left(\iota_{1}, \ldots, \iota_{k}\right) \neq(2, \ldots, 2)$. Then we obtain the stable convergence

$$
\sqrt{n}\left(V\left(Z, p_{1}^{j}, \ldots, p_{k}^{j}\right)_{t}^{n}-\rho_{p_{1}^{j}, \ldots, p_{k}^{j}}^{(n)} \int_{0}^{t}\left|\sigma_{s}\right|^{p_{+}^{j}} \mathrm{~d} s\right)_{1 \leq j \leq d} \stackrel{\mathrm{st}}{\longrightarrow} \int_{0}^{t} A_{s}^{1 / 2} \mathrm{~d} B_{s},
$$

where $B$ is a d-dimensional Brownian motion that is defined on an extension of the filtered probability space $\left(\Omega, \mathcal{F},\left(\mathcal{F}_{t}\right)_{t \geq 0}, P\right)$ and is independent of $\mathcal{F}, A$ is a $d \times d$-dimensional process given by

$$
A_{s}^{i j}=\beta_{i j}\left|\sigma_{s}\right|^{p_{+}^{i}+p_{+}^{j}}, \quad 1 \leq i, j \leq d,
$$

and the $d \times d$ matrix $\beta$ is defined in (3.11).

Proof. See Section 8.

\subsection{Discussion of assumptions}

We start our discussion again by considering the auxiliary, centered, stationary Gaussian, process

$$
G_{t}=\int_{-\infty}^{t} g(t-s) W(\mathrm{~d} s)
$$

First of all, we want to demonstrate how Theorems 1 and 2 apply for the multipower variation of the process $G$. In other words, we will give a hint how to check the conditions of these theorems.

Recall definition (2.7) of the variance function $\bar{R}$ of the increments of $G$ and note that

$$
\bar{R}(t)=E\left[\left|G_{s+t}-G_{s}\right|^{2}\right]=\int_{0}^{t} g^{2}(x) \mathrm{d} x+\int_{0}^{\infty}(g(t+x)-g(x))^{2} \mathrm{~d} x, \quad t \geq 0 .
$$

Clearly, the asymptotic behaviour of the multipower variation of the process $G$ is fully determined by the behaviour of the function $\bar{R}$ near 0 . As we deal with a continuous process $G$, it is natural to assume that $\bar{R}(t)$ behaves essentially as $t^{\alpha}$ (for some $\alpha>0$ ) near 0 (later on we will formalize this assumption). Since the case where the paths of $G$ are differentiable (a.s.) is not very interesting for us (because the consistency can be deduced by the mean value theorem), we concentrate on the region $0<\alpha<2$ (the corresponding $g(t)$ behaving as $t^{(\alpha-1) / 2}$ ).

Let us introduce a new set of assumptions that correspond to the previous discussion. These assumptions were proposed by Guyon and Leon in [22] (those authors considered the case of centered, stationary Gaussian processes $X$; this relates to the $\mathcal{B S S}$ setting with $\sigma$ constant) and the same assumptions were used in [3] and [5]. 
(A1) $\bar{R}(t)=t^{\alpha} L_{0}(t)$ for some $\alpha \in(0,2)$ and some positive slowly varying (at 0$)$ function $L_{0}$, which is continuous on $(0, \infty)$.

(A2) $\bar{R}^{\prime \prime}(t)=t^{\alpha-2} L_{2}(t)$ for some slowly varying function $L_{2}$, which is continuous on $(0, \infty)$.

(A3) There exists $b \in(0,1)$ with

$$
K=\limsup _{x \rightarrow 0} \sup _{y \in\left[x, x^{b}\right]}\left|\frac{L_{2}(y)}{L_{0}(x)}\right|<\infty .
$$

Recall that a function $L:(0, \infty) \rightarrow \mathbb{R}$ is called slowly varying at 0 when the identity

$$
\lim _{x \searrow 0} \frac{L(t x)}{L(x)}=1
$$

holds for any fixed $t>0$.

Now, note that under assumption (A1) we have, for any $j \geq 1$,

$$
\begin{aligned}
r_{n}(j) & =\operatorname{cov}\left(\frac{\Delta_{1}^{n} G}{\tau_{n}}, \frac{\Delta_{1+j}^{n} G}{\tau_{n}}\right) \\
& =\frac{\bar{R}((j+1) / n)+\bar{R}((j-1) / n)-2 \bar{R}(j / n)}{2 \bar{R}(1 / n)} \rightarrow \rho(j) \\
& =\frac{1}{2}\left((j+1)^{\alpha}-2 j^{\alpha}+(j-1)^{\alpha}\right)
\end{aligned}
$$

because $L_{0}$ is slowly varying at 0 . It is obvious that $\rho(j)$ is the correlation function of the discrete-time stationary Gaussian process $Q_{i}=B_{i}^{\alpha / 2}-B_{i-1}^{\alpha / 2}$, where $B^{\alpha / 2}$ is a fractional Brownian motion with parameter $\alpha / 2$.

Remark 7. It is easy to see that the convergence of

$$
\frac{\bar{R}((j+1) / n)+\bar{R}((j-1) / n)-2 \bar{R}(j / n)}{2 \bar{R}(1 / n)}
$$

to some real number for all $j \geq 1$ implies that there exits an $s(j)$ such that

$$
\frac{\bar{R}(j / n)}{\bar{R}(1 / n)} \rightarrow s(j) .
$$

Since the result in Theorem 4 is independent of the scale of time we use, we must have

$$
\frac{\bar{R}(j \Delta)}{\bar{R}(\Delta)} \underset{\Delta \downarrow 0}{\rightarrow} s(j)
$$

for any $\Delta$ and then $s(j k)=s(j) s(k)$; consequently $s(j)=j^{\alpha}$, for a certain $\alpha \in \mathbb{R}$. Moreover, since $(j+1)^{\alpha}-2 j^{\alpha}+(j-1)^{\alpha}$ is a covariance function, we have $0<\alpha<2$. So in the present setting, $\left(Q_{i}\right)_{i \geq 1}$, as defined in Section 3, is always a standard fractional Gaussian noise. 
As shown in [3,22] assumptions (A1)-(A3) imply that condition (3.3) holds for any $\alpha \in(0,2)$ and condition (3.9) holds for any $\alpha \in(0,3 / 2)$. Hence, Theorem 1 holds for all $\alpha \in(0,2)$ while Theorem 2 only holds for $\alpha \in(0,3 / 2)$.

Now, let us see what the conditions (A1)-(A3) mean for the memory function $g$. For simplicity, let us consider functions of the form

$$
g(x)=x^{\delta} 1_{(0,1]}(x), \quad x>0 .
$$

For such functions we readily obtain assumptions (A1) and (A2) with

$$
\alpha=2 \delta+1, \quad \delta \in\left(-\frac{1}{2}, 0\right) \cup\left(0, \frac{1}{2}\right)
$$

(the technical assumption (A3) has to be checked separately; for an example, see Section 5). Note that for $\delta=0$, for which assumption (A2) does not hold, the process $G$ is a semimartingale and the multipower variations can be treated as in [6].

Next, we discuss the assumptions of Section 4.1 for the function $g$ defined in (4.15). Recall that condition (4.4) is automatically satisfied for functions $g$ with compact support (as in (4.15)). A straightforward calculation shows that

$$
\pi^{n}((\varepsilon, \infty))=\mathrm{O}\left((n \varepsilon)^{2 \delta-1}\right)
$$

for any $\varepsilon>\frac{1}{n}$. Thus, condition (4.6) of (LLN) is satisfied (because $2 \delta-1<0$ ) and Theorem 3 is valid for all $\delta \in(-1 / 2,0) \cup(0,1 / 2)$.

Finally, we explain how to verify condition (4.8) of (CLT). Recall that $p=$ $\min _{1 \leq i \leq k, 1 \leq j \leq d}\left(p_{i}^{j}\right)$. Let $\varepsilon_{n}=n^{-\kappa}, \kappa \in(0,1)$. We readily deduce that

$$
\pi^{n}((\varepsilon, \infty))=\mathrm{O}\left(n^{\lambda(1-\kappa)}\right), \quad \lambda=2 \delta-1
$$

Thus, condition (4.8) is satisfied if

$$
\lambda<-\frac{1}{1 \wedge p} .
$$

We immediately deduce that Theorem 4 holds if

$$
\begin{aligned}
& p \geq 1: \quad \gamma>\frac{1}{2}, \quad \delta \in\left(-\frac{1}{2}, 0\right), \\
& \frac{1}{2}<p<1: \quad \gamma>\frac{1}{2 p}, \quad \delta \in\left(-\frac{1}{2}, \frac{p-1}{2 p}\right) .
\end{aligned}
$$

Remark 8. Clearly, we can deal with a larger class of functions $g$ than $g(x)=x^{\delta} 1_{(0,1]}(x)$. Assume that condition (4.4) holds. In the following we consider functions $L_{g}, L_{g^{\prime}}$, which are continuous on $(0, \infty)$ and slowly varying at 0 . We assume the following conditions:

Assumption: $g \in L^{2}((0, \infty))$ and for some $\delta \in(-1 / 2,0) \cup(0,1 / 2)$ it holds that:

(i) $g(x)=x^{\delta} L_{g}(x)$. 
(ii) $g^{\prime}(x)=x^{\delta-1} L_{g^{\prime}}(x)$ and, for any $\varepsilon>0, g^{\prime} \in L^{2}((\varepsilon, \infty))$. Moreover, $\left|g^{\prime}\right|$ is non-increasing on $(b, \infty)$ for some $b>0$.

We further assume that the function

$$
\bar{R}(t)=\int_{0}^{t} g^{2}(x) \mathrm{d} x+\int_{0}^{\infty}(g(t+x)-g(x))^{2} \mathrm{~d} x
$$

satisfies conditions (A1)-(A3) with $\alpha=2 \delta+1$.

Under these assumptions we conclude (as for the simple example $g(x)=x^{\delta} 1_{(0,1]}(x)$ ) that Theorem 3 holds for any $\delta \in(-1 / 2,0) \cup(0,1 / 2)$, and Theorem 4 holds when further

$$
\begin{aligned}
p \geq 1: \quad \gamma>\frac{1}{2}, \quad \delta \in\left(-\frac{1}{2}, 0\right), \\
\frac{1}{2}<p<1: \quad \gamma>\frac{1}{2 p}, \quad \delta \in\left(-\frac{1}{2}, \frac{p-1}{2 p}\right) .
\end{aligned}
$$

In both cases we have $Q_{i}=B_{i}^{\delta+1 / 2}-B_{i-1}^{\delta+1 / 2}, i \geq 1$.

\section{Examples}

This section discusses two examples of choice of the damping function $g$ and the associated probabilistic limit behaviour.

As above let $r$ denote the autocorrelation function of $G=\int_{-\infty}^{\cdot} g(\cdot-s) W(\mathrm{~d} s)$. Note that assumptions (A1)-(A3) could equivalently have been formulated in terms of $1-r$ rather than $\bar{R}$ (since $\left.\bar{R}(t)=2\|g\|^{2}(1-r(t))\right)$.

Suppose first that

$$
g(t)=\mathrm{e}^{-\lambda t} 1_{(0,1)}(t)
$$

with $\lambda>0$. This example (for a detailed discussion, see [12]) is a non-semimartingale case, and it can be shown that $\pi^{n} \rightarrow \pi$, with $\pi$ given by

$$
\pi=\frac{1}{1+\mathrm{e}^{-2 \lambda}} \delta_{0}+\frac{1}{1+\mathrm{e}^{2 \lambda}} \delta_{1},
$$

where $\delta_{i}$ is the Dirac measure at $i$. Moreover,

$$
V(Y, 2)_{t}^{n} \stackrel{P}{\longrightarrow}\left(1+\mathrm{e}^{-2 \lambda}\right)^{-1} \sigma_{t}^{2+}+\left(1+\mathrm{e}^{2 \lambda}\right)^{-1} \sigma_{(-1, t-1]}^{2+},
$$

where for any $a<b$

$$
\sigma_{(a, b]}^{2+}=\int_{a}^{b} \sigma_{s}^{2} \mathrm{~d} s
$$


Thus, in particular, we do not have $V(Y, 2)_{t}^{n} \stackrel{P}{\longrightarrow} \sigma_{t}^{2+}$. Note that in this example assumption (A2) is not satisfied.

Our main example is

$$
g(t)=t^{\nu-1} \mathrm{e}^{-\lambda t} 1_{(0, \infty)}(t)
$$

for $\lambda>0$ and with $v>\frac{1}{2}$. (So, for $t$ near $0, g(t)$ behaves as $t^{\delta}$ with $\delta=v-1$ ). The autocorrelation function is given by

$$
r(t)=\frac{(2 \lambda)^{2 \nu-1}}{\Gamma(2 v-1)} \mathrm{e}^{-\lambda t} \int_{0}^{\infty}(t+u)^{\nu-1} u^{\nu-1} \mathrm{e}^{-2 \lambda u} \mathrm{~d} u .
$$

It can be proved using properties of Bessel functions (see Sections 5.1-5.3 in [4]) that assumptions (A1)-(A3) are met provided that $\alpha=2 v-1 \in(0,2)$, that is, $v \in\left(\frac{1}{2}, \frac{3}{2}\right)$, and that $\rho_{p_{1}^{j}, \ldots, p_{k}^{j}}^{(n)}$ may be substituted by $\rho_{p_{1}^{j}, \ldots, p_{k}^{j}}$ in the central limit theorem provided $v \in\left(\frac{1}{2}, \frac{5}{4}\right)$. For $t \rightarrow 0$ we also have the following asymptotic equivalence

$$
1-r(t) \sim \begin{cases}2^{-2 v+1} \frac{\Gamma(3 / 2-v)}{\Gamma(v+1 / 2)}(\lambda t)^{2 v-1}+\mathrm{O}\left(t^{2}\right) & \text { for } \frac{1}{2}<v<\frac{3}{2}, \\ \frac{1}{2}(\lambda t)^{2}|\log t| & \text { for } v=\frac{3}{2}, \\ \frac{1}{4(v-3 / 2)}(\lambda t)^{2}+\mathrm{O}\left(t^{2 v-1}\right) & \text { for } \frac{3}{2}<v .\end{cases}
$$

Remark 9. So for $\frac{3}{2}<v \leq 2$ the autocorrelation function is twice differentiable at 0 and consequently $Y$ has continuously differentiable sample paths, while for $\frac{1}{2}<v \leq \frac{3}{2}$ the sample paths are Lipschitz of order $\lambda$ for every $0<\lambda<v-\frac{1}{2}$ (cf. [21], Section 9.2).

\section{An application}

Let us consider the realised variation ratio (RVR) defined for a stochastic process $X$ as

$$
R V R_{t}^{n}:=\frac{(\pi / 2) V(X, 1,1)_{t}^{n}}{V(X, 2,0)_{t}^{n}}
$$

The RVR is of interest as a diagnostic tool concerning the nature of empirical processes.

In particular, it can be used to test the hypothesis that such a process is a Brownian semimartingale (with a non-trivial local martingale component) against the possibility that it is of this type plus a jump process, see [17,26] (some related work is discussed in [34]). If a jump component is present, then the limit of $R V R_{t}^{n}$ is smaller than 1.

However, in the course of the turbulence project, mentioned earlier, when calculating the RVR for an extensive high-quality data set from atmospheric turbulence it turned out that the values of RVR were consistently higher than 1 . The wish to understand this phenomenon has been a strong motivation for the theoretical developments described in this paper. As a consequence 
of Theorem 3, we obtain the following probability limit result for the realised variation ratio of $\mathcal{B S S}$ processes:

$$
R V R_{t}^{n}-\psi\left(r_{n}(1)\right) \stackrel{\text { ucp }}{\longrightarrow} 0
$$

where

$$
\psi(\rho)=\sqrt{1-\rho^{2}}+\rho \arcsin \rho,
$$

which equals $\frac{\pi}{2} \mathrm{E}\{|U V|\}$ of two standard normal variables $U$ and $V$ with correlation $\rho$.

Moreover, we have that

$$
\begin{aligned}
\sqrt{n}\left(R V R_{t}^{n}-\psi\left(r_{n}(1)\right)\right)= & \sqrt{n}\left(\frac{(\pi / 2) V(Y, 1,1)_{t}^{n}-\psi\left(r_{n}(1)\right) \int_{0}^{t} \sigma_{s}^{2} \mathrm{~d} s}{\int_{0}^{t} \sigma_{s}^{2} \mathrm{~d} s}\right) \\
& -\sqrt{n} R V R_{t}^{n}\left(\frac{V(Y, 2,0)_{t}^{n}-\int_{0}^{t} \sigma_{s}^{2} \mathrm{~d} s}{\int_{0}^{t} \sigma_{s}^{2} \mathrm{~d} s}\right)
\end{aligned}
$$

so, if the parameter $\alpha \in(0,1)$, by applying Theorems 3 and 4 , we obtain

$$
\sqrt{n}\left(R V R_{t}^{n}-\psi\left(r_{n}(1)\right)\right) \stackrel{\mathrm{st}}{\longrightarrow}\left(\frac{\pi}{2},-\psi(\rho(1))\right) \beta^{1 / 2} \frac{\int_{0}^{t} \sigma_{s}^{2} \mathrm{~d} B_{s}}{\int_{0}^{t} \sigma_{s}^{2} \mathrm{~d} s},
$$

where $\psi$ is as above and the matrix $\beta$ is given in Theorem 2. Specifically, we find $\beta=$ $\left(\beta_{i j}\right)_{1 \leq i, j \leq 2}$, where

$$
\begin{aligned}
& \beta_{11}=\lim _{n \rightarrow \infty} n \operatorname{var}\left(V_{Q}(1,1)_{1}^{n}\right), \\
& \beta_{22}=\lim _{n \rightarrow \infty} n \operatorname{var}\left(V_{Q}(2,0)_{1}^{n}\right), \\
& \beta_{12}=\lim _{n \rightarrow \infty} n \operatorname{cov}\left(V_{Q}(2,0)_{1}^{n}, V_{Q}(1,1)_{1}^{n}\right)
\end{aligned}
$$

with $Q$ as defined in Theorem 2. Thus, we obtain

$$
\beta_{22}=\operatorname{var}\left(Q_{1}^{2}\right)+2 \sum_{k=1}^{\infty} \operatorname{cov}\left(Q_{1}^{2}, Q_{1+k}^{2}\right)=2+4 \sum_{k=1}^{\infty} \rho^{2}(k) .
$$

Similarly, we have that

$$
\beta_{12}=2 \operatorname{cov}\left(Q_{1}^{2},\left|Q_{1}\right|\left|Q_{2}\right|\right)+2 \sum_{k=1}^{\infty} \operatorname{cov}\left(Q_{1}^{2},\left|Q_{1+k}\right|\left|Q_{2+k}\right|\right)
$$


Then, if we write $E\left[\left|X_{1}^{2} X_{2} X_{3}\right|\right]:=h\left(\rho_{12}, \rho_{13}, \rho_{23}\right)$, where $X_{1}, X_{2}$ and $X_{3}$ are standard normal with $\operatorname{cov}\left(X_{i}, X_{j}\right)=\rho_{i j}$, we have

$$
\beta_{12}=\left(h(1, \rho(1), \rho(1))-\frac{2}{\pi} \psi(\rho(1))\right)+2 \sum_{k=1}^{\infty}\left(h(\rho(k), \rho(k+1), \rho(1))-\frac{2}{\pi} \psi(\rho(1))\right) .
$$

To compute the latter, we may use the following formula:

$$
h\left(\rho_{12}, \rho_{13}, \rho_{23}\right)=\frac{2}{\pi}\left(\sqrt{1-\rho_{23}^{2}}\left(1+\rho_{12}^{2}+\rho_{13}^{2}\right)+\left(\rho_{23}+2 \rho_{12} \rho_{13}\right) \arcsin \left(\rho_{23}\right)\right) ;
$$

see [28]. For the remaining term we deduce

$$
\beta_{11}=\operatorname{var}\left(\left|Q_{1}\right|\left|Q_{2}\right|\right)+2 \sum_{k=1}^{\infty} \operatorname{cov}\left(\left|Q_{1}\right|\left|Q_{2}\right|,\left|Q_{1+k}\right|\left|Q_{2+k}\right|\right) .
$$

However, while there is no explicit formula available for the latter expression, it can be computed numerically.

\section{Conclusion and outlook}

In this paper we have derived convergence in probability and normal asymptotic limit results for multipower variations of processes $Y$ that, up to a drift-like term, has the form

$$
Y_{t}=\int_{-\infty}^{t} g(t-s) \sigma_{s} W(\mathrm{~d} s)
$$

A key type of example has $g(t)$ behaving as $t^{\delta}$ for $t \downarrow 0$ and $\delta \in\left(-\frac{1}{2}, \frac{1}{2}\right) \backslash\{0\}$. In those instances, $Y$ is not a semimartingale and the limit theory of multipower variation developed for semimartingales does not suffice to derive the desired kind of limit results. The basic tool we establish and apply for this is a normal central limit theorem for triangular arrays of dependent Gaussian variables. As a case of some special interest for applications, particularly in turbulence, the central limit behaviour of the realised variation ratio, that is, the ratio of bipower variation to quadratic variation, is briefly discussed. Some specific examples of choice of $g$ are also considered.

The turbulence context concerns time-wise observations of velocities at a single location $x$ in space. More generally, it would be of interest to develop the theory of multipower variation corresponding to a setting where velocities are observed along a curve $\tau$ in space-time. More specifically, suppose that velocity $Y_{t}(x)$ at position $x$ and time $t$ is defined by

$$
Y_{t}(x)=\int_{A+(x, t)} g(t-s, x-\xi) \sigma_{s}(\xi) W(\mathrm{~d} \xi \mathrm{d} s),
$$

where $W$ denotes white noise, $\sigma_{t}(x)$ is a positive stationary random field on $\mathbb{R}^{2}, g$ is a deterministic damping function and $A$ is a subset of space-time involving only points with negative time 
coordinates. Then, with the curve $\tau$ parametrized as $\tau(w)=(x(w), t(w))$, say, the problem is to study multipower variations of the process $X$ defined as

$$
X_{w}=\int_{A+\tau(w)} g(t(w)-s, x(w)-\xi) \sigma_{s}(\xi) W(\mathrm{~d} \xi \mathrm{d} s) .
$$

Among the questions that this raises is that of proper definition of filtrations.

In another direction it would be of interest to extend the results of this paper to power and multipower variations of higher-order differences of $Y$. In particular, this might yield normal central limit theorems for the whole range of values of $\delta$ and it could also lead to more robustness against drift processes. For some recent work on quadratic variation of higher-order differences, see $[18,19]$ and references given there.

\section{Proofs}

As our proofs are rather long and technical, let us briefly outline the scheme. The proofs basically consist of four main steps:

(i) First, we provide a Wiener chaos decomposition for the functional $V\left(p_{1}^{j}, \ldots, p_{k}^{j}\right)_{t}^{n}, j=$ $1, \ldots, d$, that appears in Theorems 1 and 2 (see Section 8.3).

(ii) In a second step, we prove the weak law of large numbers and the central limit theorem for the normalized version of $\left(V\left(p_{1}^{j}, \ldots, p_{k}^{j}\right)_{t}^{n}\right)_{1 \leq j \leq d}$, using its Wiener chaos decomposition and recent techniques from Malliavin calculus derived in [30-32] (Sections 8.4 and 8.5).

(iii) In order to prove Theorems 3 and 4, we first show that the true increments $\Delta_{i}^{n} Y$ can be replaced by the quantity $\sigma_{(i-1) / n} \Delta_{i}^{n} G$ without changing the asymptotic limits (see (8.18) and (8.26)). For this step, the conditions (4.6) and (4.8) on the measure $\pi^{n}$ are absolutely crucial.

(iv) In the last step, we apply the following blocking technique: We divide the interval $[0, t]$ into big sub-blocks (whose lengths still converge to 0 ) and freeze the volatility process $\sigma$ at the beginning of each sub-block. Then Theorem 4 (respectively, Theorem 3 ) follows from Theorem 2 (respectively, Theorem 1) applied to the family of functionals $\left(V\left(G, p_{1}^{j}, \ldots, p_{k}^{j}\right)_{t}^{n}\right)_{1 \leq j \leq d}$ and the properties of stable convergence.

Below, all positive constants (which do not depend on $n$ ) are denoted by $C$, although they might change from line to line.

\subsection{Some elements of Malliavin calculus}

Before we proceed with the proofs of the main results, we review the basic concepts of the Wiener chaos expansion. Consider a complete probability space $(\Omega, \mathcal{F}, P)$ and a subspace $\mathcal{H}_{1}$ of $L^{2}(\Omega, \mathcal{F}, P)$ whose elements are zero-mean Gaussian random variables. Let $\mathbb{H}$ be a separable Hilbert space with scalar product denoted by $\langle\cdot, \cdot\rangle_{\mathbb{H}}$ and norm $\|\cdot\|_{\mathbb{H}}$. We will assume that there is an isometry

$$
\begin{aligned}
W: \mathbb{H} & \rightarrow \mathcal{H}_{1}, \\
h & \mapsto W(h)
\end{aligned}
$$


in the sense that

$$
E\left[W\left(h_{1}\right) W\left(h_{2}\right)\right]=\left\langle h_{1}, h_{2}\right\rangle_{\mathbb{H}} \cdot
$$

It is easy to see that this map has to be linear.

For any $m \geq 2$, we denote by $\mathcal{H}_{m}$ the $m$ th Wiener chaos, that is, the closed subspace of $L^{2}(\Omega, \mathcal{F}, P)$ generated by the random variables $H_{m}(X)$, where $X \in \mathcal{H}_{1}, E\left[X^{2}\right]=1$ and $H_{m}$ is the $m$ th Hermite polynomial, that is, $H_{0}(x)=1$ and $H_{m}(x)=(-1)^{m} \mathrm{e}^{x^{2} / 2} \frac{\mathrm{d}^{m}}{\mathrm{~d} x^{m}}\left(\mathrm{e}^{-x^{2} / 2}\right)$.

We denote by

$$
I_{m}: \mathbb{H}^{\odot m} \rightarrow \mathcal{H}_{m}
$$

the isometry between the symmetric tensor product $\mathbb{H}^{\odot m}$, equipped with the norm $\sqrt{m !}\|\cdot\|_{\mathbb{H} \otimes m}$, and the $m$ th chaos $\mathcal{H}_{m}$; see Section 1.1.1 in [29] for its definition. For $h \in \mathbb{H}^{\otimes m}$, we set $I_{m}(h):=$ $I_{m}(\tilde{h})$, where $\tilde{h}$ is the symmetrization of $h$. For any $g \in \mathbb{H}^{\otimes m}, h \in \mathbb{H}^{\otimes n}, n, m \geq 0$, it holds that

$$
E\left[I_{m}(g) I_{n}(h)\right]=\delta_{m n} m !\langle\tilde{g}, \tilde{h}\rangle_{\mathbb{H} \otimes m} .
$$

For any $h=h_{1} \otimes \cdots \otimes h_{m}$ and $g=g_{1} \otimes \cdots \otimes g_{m} \in \mathbb{H}^{\otimes m}$, we define the $p$ th contraction of $h$ and $g$, denoted by $h \otimes_{p} g$, as the element of $\mathbb{H}^{\otimes 2(m-p)}$ given by

$$
h \otimes_{p} g=\left\langle h_{1}, g_{1}\right\rangle_{\mathbb{H}} \cdots\left\langle h_{p}, g_{p}\right\rangle_{\mathbb{H}} h_{p+1} \otimes \cdots \otimes h_{m} \otimes g_{p+1} \otimes \cdots \otimes g_{m} .
$$

This definition can be extended by linearity to any element of $\mathbb{H}^{\otimes m}$.

Now, let $\mathcal{G}$ be the $\sigma$-field generated by the random variables $\{W(h) \mid h \in \mathbb{H}\}$. Any squareintegrable random variable $F \in L^{2}(\Omega, \mathcal{G}, P)$ has a unique chaos decomposition

$$
F=\sum_{m=0}^{\infty} I_{m}\left(h_{m}\right),
$$

where $h_{m} \in \mathbb{H}^{\odot m}$ (see [29] for more details).

Finally, we adapt the theory of Wiener chaos expansion to the set up of Section 3. Let $\mathcal{G}$ be the $\sigma$-field generated by the random variables $\left(X_{i, n}\right)_{n \geq 1,1 \leq i \leq[n t]}$ and $\mathcal{H}_{1}$ be the first Wiener chaos associated with $\left(X_{i, n}\right)_{n \geq 1,1 \leq i \leq[n t]}$, that is, the closed subspace of $L^{2}(\Omega, \mathcal{G}, P)$ generated by the random variables $\left(X_{i, n}\right)_{n \geq 1,1 \leq i \leq[n t]}$. Notice that $\mathcal{H}_{1}$ can be seen as a separable Hilbert

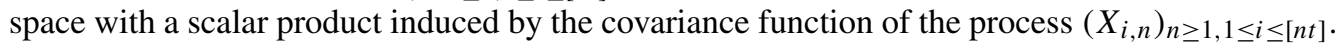
This means we can apply the above theory of Wiener chaos expansion with the canonical Hilbert space $\mathbb{H}=\mathcal{H}_{1}$. Denote by $\mathcal{H}_{m}$ the $m$ th Wiener chaos associated with the triangular array $\left(X_{i, n}\right)_{n \geq 1,1 \leq i \leq[n t]}$ and by $I_{m}$ the corresponding linear isometry between the symmetric tensor product $\mathcal{H}_{1}^{\odot m}$ (equipped with the norm $\sqrt{m !}\|\cdot\|_{\mathcal{H}_{1}^{\otimes m}}$ ) and the $m$ th Wiener chaos.

\subsection{Preliminary results}

First of all, let us note that w.l.o.g. the volatility process $\sigma$ can be assumed to be bounded on compact intervals because $\sigma$ is cadlag and it is integrated with respect to $W$. This follows by a 
standard localization procedure presented in [6]. Furthermore, the process $F_{t}$, defined by (4.4), is continuous, because $\sigma$ is cadlag. Hence, $F_{t}$ is locally bounded and can be assumed to be bounded on compact intervals w.l.o.g. by the same localization procedure.

Next we establish three lemmas.

Lemma 1. Under assumption (4.4), it holds that

$$
E\left[\left|\Delta_{i}^{n} Y\right|^{p}\right] \leq C_{p} \tau_{n}^{p}, \quad i=0, \ldots,[n t],
$$

for all $p>0$.

Proof. Recall that $g^{\prime}$ is non-increasing on $(b, \infty)$ for some $b>0$. Assume w.l.o.g. that $b>1$. Observe the decomposition

$$
\Delta_{i}^{n} Y=\int_{(i-1) / n}^{i / n} g\left(\frac{i}{n}-s\right) \sigma_{s} W(\mathrm{~d} s)+\int_{-\infty}^{(i-1) / n}\left(g\left(\frac{i}{n}-s\right)-g\left(\frac{i-1}{n}-s\right)\right) \sigma_{s} W(\mathrm{~d} s) .
$$

Since $\sigma$ is bounded on compact intervals, we deduce by Burkholder's inequality

$$
E\left[\left|\Delta_{i}^{n} Y\right|^{p}\right] \leq C_{p}\left(\tau_{n}^{p}+E\left(\int_{0}^{\infty}\left(g\left(\frac{1}{n}+s\right)-g(s)\right)^{2} \sigma_{(i-1) / n-s}^{2} \mathrm{~d} s\right)^{p / 2}\right) .
$$

We immediately obtain the estimates

$$
\begin{aligned}
& \int_{0}^{1}\left(g\left(\frac{1}{n}+s\right)-g(s)\right)^{2} \sigma_{(i-1) / n-s}^{2} \mathrm{~d} s \leq C \tau_{n}^{2}, \\
& \int_{1}^{b}\left(g\left(\frac{1}{n}+s\right)-g(s)\right)^{2} \sigma_{(i-1) / n-s}^{2} \mathrm{~d} s \leq \frac{C}{n^{2}},
\end{aligned}
$$

because $g^{\prime}$ is continuous on $(0, \infty)$ and $\sigma$ is bounded on compact intervals. On the other hand, since $g^{\prime}$ is non-increasing on $(b, \infty)$, we get

$$
\int_{b}^{\infty}\left(g\left(\frac{1}{n}+s\right)-g(s)\right)^{2} \sigma_{(i-1) / n-s}^{2} \mathrm{~d} s \leq \frac{F_{(i-1) / n}}{n^{2}} .
$$

The boundedness of the process $F$ implies (8.1).

Next, for any stochastic process $f$ and any $s>0$, we define the (possibly infinite) measure (recall that $g(x):=0$ for $x \leq 0$ )

$$
\pi_{f, s}^{n}(A)=\frac{E \int_{A}(g(x-1 / n)-g(x))^{2} f_{s-x}^{2} \mathrm{~d} x}{\int_{0}^{\infty}(g(x-1 / n)-g(x))^{2} \mathrm{~d} x}, \quad A \in \mathcal{B}(\mathbb{R}) .
$$


Lemma 2. Under assumption (4.4), it holds that

$$
\sup _{s \in[0, t]} \pi_{\sigma, s}^{n}((\varepsilon, \infty)) \leq C \pi^{n}((\varepsilon, \infty))
$$

for any $\varepsilon>0$, where $\pi^{n}$ is given by (4.5).

Proof. Recall again that $\left|g^{\prime}\right|$ is non-increasing on $(b, \infty)$ for some $b>0$, and assume w.l.o.g. that $b>\varepsilon$. Since the processes $\sigma$ and $F$ are bounded we deduce exactly as in the previous proof that

$$
\begin{aligned}
& \int_{\varepsilon}^{\infty}\left(g\left(x-\frac{1}{n}\right)-g(x)\right)^{2} \sigma_{s-x}^{2} \mathrm{~d} x \\
& \quad=\int_{\varepsilon}^{b}\left(g\left(x-\frac{1}{n}\right)-g(x)\right)^{2} \sigma_{s-x}^{2} \mathrm{~d} x+\int_{b}^{\infty}\left(g\left(x-\frac{1}{n}\right)-g(x)\right)^{2} \sigma_{s-x}^{2} \mathrm{~d} x \\
& \quad \leq C\left(\int_{\varepsilon}^{\infty}\left(g\left(x-\frac{1}{n}\right)-g(x)\right)^{2} \mathrm{~d} x+n^{-2}\right) .
\end{aligned}
$$

This completes the proof of Lemma 2.

Finally, we present the following technical lemma.

Lemma 3. Under the assumption (CLT), there exists a number $l \geq 1$ and positive sequences $\varepsilon_{n}^{(j)} \rightarrow 0, j=1, \ldots, l$, such that $0<\varepsilon_{n}^{(1)}<\cdots<\varepsilon_{n}^{(l)}$ and

$$
\begin{aligned}
& \varepsilon_{n}^{(1)}=\mathrm{o}\left(n^{-1 /(2 \gamma(p \wedge 1))}\right), \quad \pi^{n}\left(\left(\varepsilon_{n}^{(l)}, \infty\right)\right)=\mathrm{o}\left(n^{-1 /(p \wedge 1)}\right), \\
& \left(\varepsilon_{n}^{(j+1)}\right)^{2 \gamma} \pi^{n}\left(\left(\varepsilon_{n}^{(j)}, \infty\right)\right)=\mathrm{o}\left(n^{-1 /(p \wedge 1)}\right), \quad j=1, \ldots, l-1,
\end{aligned}
$$

where $p=\min _{1 \leq i \leq k, 1 \leq j \leq d}\left(p_{i}^{j}\right)$.

Proof. Assume first that $p \geq 1$. Recall that $\gamma>1 / 2$. Set $\varepsilon_{n}^{(j)}=n^{-\kappa_{j}}, j=1, \ldots, l$, with $1>$ $\kappa_{1}>\cdots>\kappa_{l}>0$. The condition $\pi^{n}\left(\left(\varepsilon_{n}^{(j)}, \infty\right)\right)=\mathrm{O}\left(n^{\lambda\left(1-\kappa_{j}\right)}\right)$ for some $\lambda<-1$, presented in (4.8), implies that conditions (8.4) and (8.5) are satisfied if we find $1>\kappa_{1}>\cdots>\kappa_{l}>0$ such that

$$
\begin{array}{rlrl}
\kappa_{1} & >\frac{1}{2 \gamma}, & \kappa_{l}<1+\frac{1}{\lambda}, \\
(1+\lambda)-\kappa_{j} \lambda-2 \kappa_{j+1} \gamma & <0, \quad 1 \leq j \leq l-1 .
\end{array}
$$

From the first and the last inequality, we deduce by induction that

$$
\frac{1}{2 \gamma}<\kappa_{1}<\frac{1+\lambda}{\lambda} \sum_{i=0}^{l-1}\left(-\frac{2 \gamma}{\lambda}\right)^{i}+\left(-\frac{2 \gamma}{\lambda}\right)^{l} \kappa_{l}
$$


must hold.

When $2 \gamma \geq-\lambda$, the term on the right-hand side converges to $\infty$ as $l \rightarrow \infty$. In that case it is easy to find constants $1>\kappa_{1}>\cdots>\kappa_{l}>0$ such that (8.4) and (8.5) are satisfied.

When $2 \gamma<-\lambda$, the limit of $\frac{1+\lambda}{\lambda} \sum_{i=0}^{l-1}\left(-\frac{2 \gamma}{\lambda}\right)^{i}$ is $\frac{1+\lambda}{\lambda+2 \gamma}($ as $l \rightarrow \infty)$ and the restriction on $\kappa_{1}$ becomes

$$
\frac{1}{2 \gamma}<\kappa_{1}<\frac{1+\lambda}{\lambda+2 \gamma}
$$

Notice that $\frac{1}{2 \gamma}<\frac{1+\lambda}{\lambda+2 \gamma}$ because $\gamma>1 / 2$. The existence of the positive powers $\kappa_{j}, j=2, \ldots, l$ that satisfy the original inequality follows by an induction argument.

Assume now that $p<1$. Recall that $\gamma$ must satisfy

$$
\gamma>\frac{1}{2 p}
$$

and $\lambda<-\frac{1}{p}$. Again the conditions (8.4) and (8.5) are satisfied if we find $1>\kappa_{1}>\cdots>\kappa_{l}>0$ such that

$$
\begin{aligned}
\kappa_{1}>\frac{1}{2 \gamma p}, \quad \kappa_{l}<\frac{1+\lambda p}{\lambda p}, \\
\left(\frac{1}{p}+\lambda\right)-\kappa_{j} \lambda-2 \kappa_{j+1} \gamma<0, \quad 1 \leq j \leq l-1 .
\end{aligned}
$$

Notice that the second inequality has solutions because $\lambda<-\frac{1}{p}$. Moreover, we deduce as above that the inequality

$$
\frac{1}{2 \gamma p}<\kappa_{1}<\frac{1 / p+\lambda}{\lambda} \sum_{i=0}^{l-1}\left(-\frac{2 \gamma}{\lambda}\right)^{i}+\left(-\frac{2 \gamma}{\lambda}\right)^{l} \kappa_{l}
$$

must hold. Again the more complicated case is $2 \gamma<-\lambda$. By letting $l \rightarrow \infty$, the restriction on $\kappa_{1}$ becomes

$$
\frac{1}{2 \gamma p}<\kappa_{1}<\frac{1 / p+\lambda}{\lambda+2 \gamma}
$$

Note that $\frac{1}{2 \gamma p}<\frac{1 / p+\lambda}{\lambda+2 \gamma}$ because $\gamma>\frac{1}{2 p}$. As before, the existence of the positive powers $\kappa_{j}$, $j=2, \ldots, l$ that satisfy the original inequality follows by an induction argument.

\subsection{Some notation}

In this subsection we introduce various notation connected to the Wiener chaos expansion for the functionals $V\left(p_{1}^{j}, \ldots, p_{k}^{j}\right)_{t}^{n}, j=1, \ldots, d$, and present some first convergence results. 
Recall that the covariance matrix of $\left(X_{i, n}, \ldots, X_{i+l, n}\right)$ is invertible for any $l \geq 1$ and $n \geq$ 1. Let $X_{i}^{n}(1), \ldots, X_{i}^{n}(k)$ be an i.i.d. $N(0,1)$ sequence that spans the same linear space as $X_{i, n}, \ldots, X_{i+k-1, n}$ (such a sequence can be constructed by the Gram-Schmidt method). Thus, it has the representation

$$
X_{i}^{n}(j)=\sum_{l=1}^{k} a_{l j}^{n} X_{i+l-1, n}, \quad j=1, \ldots, k,
$$

for some real numbers $a_{l j}^{n}$. Note that

$$
\left|a_{l j}^{n}\right| \leq C
$$

for all $l, j, n$, because $E\left[X_{i, n}^{2}\right]=1$ for all $i, n$.

For any $1 \leq j \leq d$, we obtain the Wiener chaos representation

$$
V\left(p_{1}^{j}, \ldots, p_{k}^{j}\right)_{t}^{n}-\rho_{p_{1}^{j}, \ldots, p_{k}^{j}}^{(n)} t=\sum_{m=2}^{\infty} I_{m}\left(\frac{1}{n} \sum_{i=1}^{[n t]} f_{m, j}^{n}(i)\right)+\mathrm{O}_{p}\left(n^{-1}\right),
$$

where the $f_{m, j}^{n}(i) \in \mathbb{H}^{\odot m}$ are given by

$$
f_{m, j}^{n}(i)=\sum_{k_{l} \in\{1, \ldots, k\}} c_{k_{1}, \ldots, k_{m}}^{n}(j) X_{i}^{n}\left(k_{1}\right) \otimes \cdots \otimes X_{i}^{n}\left(k_{m}\right)
$$

for some coefficients $c_{k_{1}, \ldots, k_{m}}^{n}(j)$. We set

$$
c_{m}^{n}(j)=\left\|f_{m, j}^{n}(i)\right\|_{\mathbb{H} \otimes m}^{2}=\sum_{k_{l} \in\{1, \ldots, k\}}\left|c_{k_{1}, \ldots, k_{m}}^{n}(j)\right|^{2} .
$$

Note that

$$
\operatorname{var}\left(\left|X_{i, n}\right|^{p_{1}^{j}} \ldots\left|X_{i+k-1, n}\right|^{p_{k}^{j}}\right)=\sum_{m=2}^{\infty} m ! c_{m}^{n}(j)<C
$$

for all $n, j$, because $E\left[X_{i, n}^{2}\right]=1$ for all $i, n$. Finally, when $f_{m, j}^{n}(i), c_{k_{1}, \ldots, k_{m}}^{n}(j)$ and $c_{m}^{n}(j)$ correspond to some particular choice of powers $p_{1}, \ldots, p_{k}$, we use the notation $f_{m}^{n}(i), c_{k_{1}, \ldots, k_{m}}^{n}$ and $c_{m}^{n}$.

Now assume that the assumptions (3.8) and (3.9) of Theorem 2 hold. Since $a_{l j}^{n}$ in (8.6) is a continuous function of $r(1), \ldots, r_{n}(k-1)$ and the Gaussian process $Q$ is non-degenerate, we have that

$$
a_{l j}^{n} \rightarrow a_{l j}
$$

and the sequence $Q_{i}(1), \ldots, Q_{i}(k)$ given by

$$
Q_{i}(j)=\sum_{l=1}^{k} a_{l j} Q_{i+l-1}, \quad j=1, \ldots, k,
$$


is an i.i.d. $N(0,1)$ sequence. Now, let us associate $f_{m, j}(i), c_{k_{1}, \ldots, k_{m}}(j)$ and $c_{m}(j)$ with the functional $V_{Q}\left(p_{1}^{j}, \ldots, p_{k}^{j}\right)_{t}^{n}-\rho_{p_{1}^{j}, \ldots, p_{k}^{j}} t$, where

$$
\rho_{p_{1}^{j}, \ldots, p_{k}^{j}}=E\left[\left|Q_{1}\right|^{p_{1}^{j}} \ldots\left|Q_{k}\right|^{p_{k}^{j}}\right],
$$

by (8.7), (8.8) and (8.9). By a repeated application of the multiplication formula (see [29]), we know that $c_{k_{1}, \ldots, k_{m}}^{n}(j)$ is a continuous function of $r_{n}(1), \ldots, r_{n}(k-1)$. Since $r_{n}(j) \rightarrow \rho(j)$ we obtain

$$
\begin{gathered}
c_{k_{1}, \ldots, k_{m}}^{n}(j) \rightarrow c_{k_{1}, \ldots, k_{m}}(j), \quad c_{m}^{n}(j) \rightarrow c_{m}(j), \\
\left\langle f_{m, j_{1}}^{n}(i), f_{m, j_{2}}^{n}(i+l)\right\rangle_{\mathbb{H} \otimes m} \rightarrow\left\langle f_{m, j_{1}}(i), f_{m, j_{2}}(i+l)\right\rangle_{\mathbb{H}} \otimes m, \\
\operatorname{cov}\left(\left|X_{i, n}\right|^{p_{1}^{j_{1}}} \cdots\left|X_{i+k-1, n}\right|^{p_{k}^{j_{1}}},\left|X_{i, n}\right|^{p_{1}^{j_{2}}} \cdots\left|X_{i+k-1, n}\right|^{p_{k}^{j_{2}}}\right) \\
=\sum_{m=2}^{\infty} m !\left\langle f_{m, j_{1}}^{n}(1), f_{m, j_{2}}^{n}(1)\right\rangle_{\mathbb{H} \otimes m} \\
\rightarrow \operatorname{cov}\left(\left|Q_{i}\right|^{p_{1}^{j_{1}} \cdots \mid}\left|Q_{i+k-1}\right|^{p_{k}^{j_{1}}},\left|X_{i, n}\right|^{\left.p_{1}^{j_{2}} \cdots\left|Q_{i+k-1}\right|_{k}^{j_{2}}\right)}\right. \\
=\sum_{m=2}^{\infty} m !\left\langle f_{m, j_{1}}(1), f_{m, j_{2}}(1)\right\rangle_{\mathbb{H} \otimes m} .
\end{gathered}
$$

\subsection{Proof of Theorems 1 and 3}

Proof of Theorems 1. Since $V\left(p_{1}, \ldots, p_{k}\right)_{t}^{n}$ is increasing in $t$ and the process $\rho_{p_{1}, \ldots, p_{k}}^{(n)} t$ is continuous in $t$, it is sufficient to prove $V\left(p_{1}, \ldots, p_{k}\right)_{t}^{n}-\rho_{p_{1}, \ldots, p_{k}}^{(n)} \stackrel{P}{\longrightarrow} 0$ for a fixed $t>0$.

Note that

$$
\begin{aligned}
\left|\left\langle f_{m}^{n}(1), f_{m}^{n}(1+l)\right\rangle_{\mathbb{H} \otimes m}\right| & \leq c_{m}^{n}, \\
\left|\left\langle f_{m}^{n}(1), f_{m}^{n}(1+l)\right\rangle_{\mathbb{H} \otimes m}\right| & \leq c_{m}^{n} C^{m}\left(\left|r_{n}(l)\right|^{m}+\cdots+\left|r_{n}(l-k+1)\right|^{m}\right),
\end{aligned}
$$

where the bounds are not comparable. Now, due to assumption (3.3), $\mathrm{r}(j) \rightarrow 0$ as $j \rightarrow \infty$. Thus, there exists an $H$ such that $\left|C \mathrm{r}^{1 / 2}(j-k+1)\right|<1$ for $j \geq H$ (for any fixed $C$ ). By (8.15) we have (for any $m \geq 2$ )

$$
\begin{aligned}
\sum_{l=1}^{n-1}\left|\left\langle f_{m}^{n}(1), f_{m}^{n}(1+l)\right\rangle_{\mathbb{H}^{\otimes m}}\right| & \leq C\left(H c_{m}^{n}+\sum_{l=H}^{n-1}\left|\left\langle f_{m}^{n}(1), f_{m}^{n}(1+l)\right\rangle_{\mathbb{H}^{\otimes m}}\right|\right) \\
& \leq C c_{m}^{n}\left(H+\sum_{l=H}^{n-1}\left(C\left|r_{n}(l)\right|\right)^{2}\right) \leq C c_{m}^{n} \sum_{l=1}^{n-1} \mathrm{r}(l) .
\end{aligned}
$$


Hence,

$$
\operatorname{var}\left(V\left(p_{1}, \ldots, p_{k}\right)_{t}^{n}\right) \leq \frac{C}{n} \sum_{m=2}^{\infty} m ! c_{m}^{n}\left(1+\sum_{l=1}^{n-1} \mathrm{r}(l)\right)
$$

The latter converges to 0 due to (8.10) and assumption (3.3).

Proof of Theorem 3. Assume first that $Z_{1}=0$. Recall that

$$
E\left[\left|\Delta_{i}^{n} Y\right|^{q}\right] \leq C \tau_{n}^{q}, \quad E\left[\left|\Delta_{i}^{n} G\right|^{q}\right] \leq C \tau_{n}^{q}
$$

for any $q \geq 0$, due to Lemma 1 .

We assume for simplicity that $k=1, p_{1}=p$. The general case can be proved in a similar manner by (8.17) and an application of the Hölder inequality.

Since $V(Y, p)_{t}^{n}$ is increasing in $t$ and the limit process is continuous in $t$, it suffices to prove the pointwise convergence $V(Y, p)_{t}^{n} \stackrel{P}{\longrightarrow} \mu_{p} \int_{0}^{t}\left|\sigma_{s}\right|^{p} \mathrm{~d} s$. For any $l \leq n$, we have

$$
V(Y, p)_{t}^{n}-\mu_{p} \int_{0}^{t}\left|\sigma_{s}\right|^{p} \mathrm{~d} s=\frac{1}{n \tau_{n}^{p}} \sum_{i=1}^{[n t]}\left(\left|\Delta_{i}^{n} Y\right|^{p}-\left|\sigma_{(i-1) / n} \Delta_{i}^{n} G\right|^{p}\right)+R_{t}^{n, l},
$$

where

$$
\begin{aligned}
R_{t}^{n, l}= & \frac{1}{n \tau_{n}^{p}}\left(\sum_{i=1}^{[n t]}\left|\sigma_{(i-1) / n} \Delta_{i}^{n} G\right|^{p}-\sum_{j=1}^{[l t]}\left|\sigma_{(j-1) / l}\right|^{p} \sum_{i \in I_{l}(j)}\left|\Delta_{i}^{n} G\right|^{p}\right) \\
& +\frac{1}{n \tau_{n}^{p}} \sum_{j=1}^{[l t]}\left|\sigma_{(j-1) / l}\right|^{p} \sum_{i \in I_{l}(j)}\left|\Delta_{i}^{n} G\right|^{p}-\mu_{p} l^{-1} \sum_{j=1}^{[l t]}\left|\sigma_{(j-1) / l}\right|^{p} \\
& +\mu_{p}\left(l^{-1} \sum_{j=1}^{[l t]}\left|\sigma_{(j-1) / l}\right|^{p}-\int_{0}^{t}\left|\sigma_{s}\right|^{p} \mathrm{~d} s\right)
\end{aligned}
$$

and

$$
I_{l}(j)=\left\{i \mid \frac{i}{n} \in\left(\frac{j-1}{l}, \frac{j}{l}\right]\right\}, \quad j \geq 1
$$

The assumption (LLN) implies that $V(G, p)_{t}^{n} \stackrel{\text { ucp }}{\longrightarrow} \mu_{p} t$. Since $\sigma$ is cadlag and bounded on compact intervals, we deduce that

$$
\lim _{l \rightarrow \infty} \lim _{n \rightarrow \infty} P\left(\left|R_{t}^{n, l}\right|>\epsilon\right)=0
$$


for any $\epsilon>0$. Hence, we are left to prove that

$$
\frac{1}{n \tau_{n}^{p}} \sum_{i=1}^{[n t]}\left(\left|\Delta_{i}^{n} Y\right|^{p}-\left|\sigma_{(i-1) / n} \Delta_{i}^{n} G\right|^{p}\right) \stackrel{P}{\longrightarrow} 0 .
$$

By applying the inequality $\left.|| x\right|^{p}-|y|^{p}|\leq p| x-y \mid\left(|x|^{p-1}+|y|^{p-1}\right)$ for $p>1$ and $\left.|| x\right|^{p}-$ $|y|^{p}|\leq| x-\left.y\right|^{p}$ for $p \leq 1$, (8.17) and the Cauchy-Schwarz inequality, we can conclude that the above convergence follows from

$$
\frac{1}{n \tau_{n}^{2}} \sum_{i=1}^{[n t]} E\left[\left|\Delta_{i}^{n} Y-\sigma_{(i-1) / n} \Delta_{i}^{n} G\right|^{2}\right] \rightarrow 0
$$

Observe the decomposition

$$
\Delta_{i}^{n} Y-\sigma_{(i-1) / n} \Delta_{i}^{n} G=A_{i}^{n}+B_{i}^{n, \varepsilon}+C_{i}^{n, \varepsilon}
$$

where

$$
\begin{aligned}
A_{i}^{n}= & \int_{(i-1) / n}^{i / n} g\left(\frac{i}{n}-s\right)\left(\sigma_{s}-\sigma_{(i-1) / n}\right) W(\mathrm{~d} s) \\
B_{i}^{n, \varepsilon}= & \int_{(i-1) / n-\varepsilon}^{(i-1) / n}\left(g\left(\frac{i}{n}-s\right)-g\left(\frac{i-1}{n}-s\right)\right) \sigma_{s} W(\mathrm{~d} s) \\
& -\sigma_{(i-1) / n} \int_{(i-1) / n-\varepsilon}^{(i-1) / n}\left(g\left(\frac{i}{n}-s\right)-g\left(\frac{i-1}{n}-s\right)\right) W(\mathrm{~d} s), \\
C_{i}^{n, \varepsilon}= & \int_{-\infty}^{(i-1) / n-\varepsilon}\left(g\left(\frac{i}{n}-s\right)-g\left(\frac{i-1}{n}-s\right)\right) \sigma_{s} W(\mathrm{~d} s) \\
& -\sigma_{(i-1) / n} \int_{-\infty}^{(i-1) / n-\varepsilon}\left(g\left(\frac{i}{n}-s\right)-g\left(\frac{i-1}{n}-s\right)\right) W(\mathrm{~d} s) .
\end{aligned}
$$

By Lemma 2 and the boundedness of $\sigma$ on compact intervals, we deduce

$$
\frac{1}{n \tau_{n}^{2}} \sum_{i=1}^{[n t]} E\left[\left|C_{i}^{n, \varepsilon}\right|^{2}\right] \leq C \pi^{n}((\varepsilon, \infty)),
$$

and by (4.6) we obtain that

$$
\lim _{n \rightarrow \infty} \frac{1}{n \tau_{n}^{2}} \sum_{i=1}^{[n t]} E\left[\left|C_{i}^{n, \varepsilon}\right|^{2}\right]=0 .
$$


Next, we get

$$
\frac{1}{n \tau_{n}^{2}} \sum_{i=1}^{[n t]} E\left[\left|A_{i}^{n}\right|^{2}\right] \leq \frac{C}{n \tau_{n}^{2}} E\left[\sum_{i=1}^{[n t]} \int_{(i-1) / n}^{i / n} g^{2}\left(\frac{i}{n}-s\right)\left(\sigma_{s}-\sigma_{(i-1) / n}\right)^{2} \mathrm{~d} s\right] .
$$

Set $v(s, \eta)=\sup \left\{\left|\sigma_{s}-\sigma_{r}\right|^{2}|s, r \in[-t, t]| r-s \mid, \leq \eta\right\}$. Then we obtain

$$
\frac{1}{n \tau_{n}^{2}} \sum_{i=1}^{[n t]} E\left[\left|A_{i}^{n}\right|^{2}\right] \leq \frac{1}{n} \sum_{i=1}^{[n t]} E\left[v\left(\frac{i-1}{n}, n^{-1}\right)\right]
$$

Moreover, for any $\kappa>0$, since $\sigma$ is cadlag, there exists $n$ big enough that

$$
v\left(\frac{i-1}{n}, n^{-1}\right) \leq \kappa+\left(\Delta \sigma_{(i-1) / n}\right)^{2} \mathbf{1}_{\left\{\left(\Delta \sigma_{(i-1) / n}\right)^{2} \geq \kappa\right\}}
$$

so

$$
\begin{aligned}
\frac{1}{n \tau_{n}^{2}} \sum_{i=1}^{[n t]} E\left[\left|A_{i}^{n}\right|^{2}\right] & \leq \kappa+\frac{1}{n} \sum_{i=1}^{[n t]} E\left[\left(\Delta \sigma_{(i-1) / n}\right)^{2} \mathbf{1}_{\left\{\left(\Delta \sigma_{(i-1) / n}\right)^{2} \geq \kappa\right\}}\right] \\
& \leq \kappa+E\left[\frac{1}{n} \sum_{-t \leq s \leq t}\left(\Delta \sigma_{s}\right)^{2} \mathbf{1}_{\left\{\left(\Delta \sigma_{s}\right)^{2} \geq \kappa\right\}}\right]
\end{aligned}
$$

then

$$
\lim _{n \rightarrow \infty} \frac{1}{n \tau_{n}^{2}} \sum_{i=1}^{[n t]} E\left[\left|A_{i}^{n}\right|^{2}\right] \leq \kappa
$$

and the convergence to zero follows, letting $\kappa$ tend to zero.

Finally, observe the decomposition $B_{i}^{n, \varepsilon}=B_{i}^{n, \varepsilon}(1)+B_{i}^{n, \varepsilon}(2)$ with

$$
\begin{aligned}
& B_{i}^{n, \varepsilon}(1)=\int_{(i-1) / n-\varepsilon}^{(i-1) / n}\left(g\left(\frac{i}{n}-s\right)-g\left(\frac{i-1}{n}-s\right)\right)\left(\sigma_{s}-\sigma_{(i-1) / n-\varepsilon}\right) W(\mathrm{~d} s), \\
& B_{i}^{n, \varepsilon}(2)=\left(\sigma_{(i-1) / n-\varepsilon}-\sigma_{(i-1) / n}\right) \int_{(i-1) / n-\varepsilon}^{(i-1) / n}\left(g\left(\frac{i}{n}-s\right)-g\left(\frac{i-1}{n}-s\right)\right) W(\mathrm{~d} s) .
\end{aligned}
$$

We obtain the inequalities

$$
\begin{aligned}
& \frac{1}{n \tau_{n}^{2}} \sum_{i=1}^{[n t]} E\left[\left|B_{i}^{n, \varepsilon}(1)\right|^{2}\right] \leq \frac{1}{n} \sum_{i=1}^{[n t]} E\left[v\left(\frac{i-1}{n}, \varepsilon\right)\right] \\
& \frac{1}{n \tau_{n}^{2}} \sum_{i=1}^{[n t]} E\left[\left|B_{i}^{n, \varepsilon}(2)\right|^{2}\right] \leq \frac{1}{n} \sum_{i=1}^{[n t]} E\left[v\left(\frac{i-1}{n}, \varepsilon\right)^{2}\right]^{1 / 2} .
\end{aligned}
$$


By using the same arguments as above, we have that both terms converge to zero and we obtain (8.18), which completes the proof with $Z_{1}=0$.

To prove the general case, with $Z_{1} \neq 0$, we consider, for simplicity, the case $k=2$.

Assume first that $0 \leq p_{1}, p_{2} \leq 1$. We have ||$x_{1}+\left.y_{1}\right|^{p_{1}}\left|x_{2}+y_{2}\right|^{p_{2}}-\left|y_{1}\right|^{p_{1}}\left|y_{2}\right|^{p_{2}} \mid \leq$ $C\left(\left|x_{1}\right|^{p_{1}}\left|x_{2}\right|^{p_{2}}+\left|x_{1}\right|^{p_{1}}\left|y_{2}\right|^{p_{2}}+\left|y_{1}\right|^{p_{1}}\left|x_{2}\right|^{p_{2}}\right)$. Hence we deduce

$$
\begin{aligned}
& \left|V\left(Z, p_{1}, p_{2}\right)_{t}^{n}-V\left(Y, p_{1}, p_{2}\right)_{t}^{n}\right| \\
& \quad \leq \frac{C}{n \tau_{n}^{p_{+}}} \sum_{i=1}^{[n t]-1}\left(\left|\Delta_{i}^{n} Z_{1}\right|^{p_{1}}\left|\Delta_{i+1}^{n} Z_{1}\right|^{p_{2}}+\left|\Delta_{i}^{n} Z_{1}\right|^{p_{1}}\left|\Delta_{i+1}^{n} Z_{2}\right|^{p_{2}}+\left|\Delta_{i}^{n} Z_{2}\right|^{p_{1}}\left|\Delta_{i+1}^{n} Z_{1}\right|^{p_{2}}\right),
\end{aligned}
$$

and the result follows by (4.10).

Next, assume that $p_{1} \leq p_{2}, p_{2}>1$. We deduce that

$$
\begin{aligned}
& \left|\left(V\left(Z, p_{1}, p_{2}\right)_{t}^{n}\right)^{1 / p_{2}}-\left(V\left(Y, p_{1}, p_{2}\right)_{t}^{n}\right)^{1 / p_{2}}\right| \\
& \leq C\left(\left(\frac{1}{n \tau_{n}^{p_{+}}} \sum_{i=1}^{[n t]-1}\left|\Delta_{i}^{n} Z_{1}\right|^{p_{1}}\left|\Delta_{i+1}^{n} Z_{1}\right|^{p_{2}}\right)^{1 / p_{2}}+\left(\frac{1}{n \tau_{n}^{p_{+}}} \sum_{i=1}^{[n t]-1}\left|\Delta_{i}^{n} Z_{1}\right|^{p_{1}}\left|\Delta_{i+1}^{n} Z_{2}\right|^{p_{2}}\right)^{1 / p_{2}}\right. \\
& \left.\quad+\left(\frac{1}{n \tau_{n}^{p_{+}}} \sum_{i=1}^{[n t]-1}\left|\Delta_{i}^{n} Z_{2}\right|^{p_{1}}\left|\Delta_{i+1}^{n} Z_{1}\right|^{p_{2}}\right)^{1 / p_{2}}\right)
\end{aligned}
$$

which completes the proof of Theorem 3.

\subsection{Proof of Theorems 2 and 5}

Proof of Theorem 2. We first show the weak convergence of finite-dimensional distributions and then prove the tightness of the sequence $\sqrt{n}\left(V\left(p_{1}^{j}, \ldots, p_{k}^{j}\right)_{t}^{n}-\rho_{p_{1}^{j}, \ldots, p_{k}^{j}}^{(n)} t\right)_{1 \leq j \leq d}$.

Step 1: Define the vector $Z_{n}(j)=\left(Z_{n}^{1}(j), \ldots, Z_{n}^{\mathrm{e}}(j)\right)^{T}, 1 \leq j \leq d$, by

$$
Z_{n}^{l}(j)=\frac{1}{\sqrt{n}} \sum_{i=\left[n c_{l}\right]+1}^{\left[n b_{l}\right]}\left(\left|X_{i, n}\right|^{p_{1}^{j}} \ldots\left|X_{i+k-1, n}\right|^{p_{k}^{j}}-\rho_{p_{1}^{j}, \ldots, p_{k}^{j}}^{(n)}\right),
$$

where $\left(c_{l}, b_{l}\right], l=1, \ldots, \mathrm{e}$, are disjoint intervals contained in $[0, T]$. Set $Z_{n}^{l}=\left(Z_{n}^{l}(1), \ldots\right.$, $\left.Z_{n}^{l}(d)\right), l=1, \ldots$, e. Clearly, it suffices to prove that

$$
\left(Z_{n}^{l}\right)_{1 \leq l \leq \mathrm{e}} \stackrel{\mathcal{D}}{\longrightarrow}\left(\beta^{1 / 2}\left(B_{b_{l}}-B_{c_{l}}\right)\right)_{1 \leq l \leq \mathrm{e}},
$$

where the matrix $\beta$ is given in Theorem 2. By (8.7), we have the representation

$$
Z_{n}^{l}(j)=\sum_{m=2}^{\infty} I_{k}\left(\frac{1}{\sqrt{n}} \sum_{i=\left[n c_{l}\right]+1}^{\left[n b_{l}\right]} f_{m, j}^{n}(i)\right)
$$


Set $F_{m, l}^{n}(j)=\frac{1}{\sqrt{n}} \sum_{i=\left[n c_{l}\right]+1}^{\left[n b_{l}\right]} f_{m, j}^{n}(i)$. By Theorem 2 in [5] we obtain the weak convergence of finite-dimensional distributions when we show that:

(i) For any $1 \leq l \leq \mathrm{e}, 1 \leq j \leq d$, we have

$$
\lim _{N \rightarrow \infty} \limsup _{n \rightarrow \infty} \sum_{m=N+1}^{\infty} m !\left\|F_{m, l}^{n}(j)\right\|_{\mathbb{H} \otimes k}^{2}=0 .
$$

(ii) For any $m \geq 2,1 \leq l \leq \mathrm{e}$ and $1 \leq j_{1}, j_{2} \leq d$, we have constants $C_{k, l}$ such that

$$
\lim _{n \rightarrow \infty} m !\left\langle F_{m, l}^{n}\left(j_{1}\right), F_{m, l}^{n}\left(j_{2}\right)\right\rangle_{\mathbb{H} \otimes m}=C_{m, l}\left(j_{1}, j_{2}\right)
$$

and $\sum_{m=2}^{\infty} C_{m, l}\left(j_{1}, j_{2}\right)=\beta_{j_{1}, j_{2}}\left(b_{l}-c_{l}\right)$.

(iii) For any $1 \leq l_{1} \neq l_{2} \leq \mathrm{e}$ and $1 \leq j_{1}, j_{2} \leq d$, we have

$$
\lim _{n \rightarrow \infty}\left\langle F_{m, l_{1}}^{n}\left(j_{1}\right), F_{m, l_{2}}^{n}\left(j_{2}\right)\right\rangle_{\mathbb{H} \otimes m}=0
$$

(iv) For any $m \geq 2,1 \leq l \leq \mathrm{e}, 1 \leq j \leq d$ and $p=1, \ldots, m-1$

$$
\lim _{n \rightarrow \infty}\left\|F_{m, l}^{n}(j) \otimes_{p} F_{m, l}^{n}(j)\right\|_{\mathbb{H} \otimes 2(m-p)}^{2}=0 .
$$

Note that it is sufficient to prove (i), (ii) and (iv) for $l=1, b_{l}=1$ and $a_{l}=0$. In this case we use the notation $F_{m}^{n}(j)=F_{m, 1}^{n}(j)$.

(i) and (ii) As in (8.16), we have

$$
\begin{aligned}
& m !\left\langle F_{m}^{n}\left(j_{1}\right), F_{m}^{n}\left(j_{2}\right)\right\rangle_{\mathbb{H} \otimes m} \\
& \quad=m !\left(\left\langle f_{m, j_{1}}^{n}(1), f_{m, j_{2}}^{n}(1)\right\rangle_{\mathbb{H} \otimes m}+\frac{2}{n} \sum_{l=1}^{n-1}(n-l)\left\langle f_{m, j_{1}}^{n}(1), f_{m, j_{2}}^{n}(1+l)\right\rangle_{\mathbb{H}^{\mathbb{N}} \otimes m}\right) \\
& \quad \leq C m !\left\langle f_{m, j_{1}}^{n}(1), f_{m, j_{2}}^{n}(1)\right\rangle_{\mathbb{H} \otimes m}\left(1+\sum_{l=1}^{n-1} \mathrm{r}(l)\right) .
\end{aligned}
$$

Since $\sum_{l=1}^{\infty} r(l)<\infty$, we obtain by (8.12)-(8.14) and the dominated convergence theorem

$$
\begin{aligned}
\lim _{n \rightarrow \infty} m !\left\langle F_{m, l}^{n}\left(j_{1}\right), F_{m, l}^{n}\left(j_{2}\right)\right\rangle_{\mathbb{H} \otimes m} \\
\quad=C_{m}\left(j_{1}, j_{2}\right) \\
\quad=m !\left(\left\langle f_{m, j_{1}}(1), f_{m, j_{2}}(1)\right\rangle_{\mathbb{H} \otimes m}+2 \sum_{l=1}^{\infty}\left\langle f_{m, j_{1}}^{n}(1), f_{m, j_{2}}^{n}(1+l)\right\rangle_{\mathbb{H} \otimes m}\right)
\end{aligned}
$$


and $\sum_{m=2}^{\infty} C_{m}\left(j_{1}, j_{2}\right)=\beta_{j_{1}, j_{2}}$ (notice that $\beta_{j_{1}, j_{2}}$ is finite due to the dominated convergence theorem). Hence, we deduce (ii). On the other hand, we have

$$
\limsup _{n \rightarrow \infty} \sum_{m=N+1}^{\infty} m !\left\langle f_{m, j_{1}}^{n}(1), f_{m, j_{2}}^{n}(1)\right\rangle_{\mathbb{H} \otimes m}=\sum_{m=N+1}^{\infty} m !\left\langle f_{m, j_{1}}(1), f_{m, j_{2}}(1)\right\rangle_{\mathbb{H} \otimes m}<\infty .
$$

Thus, we obtain (i) by (8.24).

(iii) W.l.o.g. consider the case $j=j_{1}=j_{2}$. For any $l_{1}<l_{2}$, as in (8.16), we have

$$
\left|\left\langle F_{m, l_{1}}^{n}(j), F_{m, l_{2}}^{n}(j)\right\rangle_{\mathbb{H} \otimes m}\right| \leq \frac{C}{n} \sum_{h=\left[n c_{l_{1}}\right]+1}^{\left[n b_{l_{1}}\right]} \sum_{i=\left[n c_{l_{2}}\right]+1}^{\left[n b_{l_{2}}\right]}\left|r_{n}^{m}(i-h)\right| .
$$

Assume w.l.o.g. that $c_{l_{1}}=0, b_{l_{1}}=c_{l_{2}}=1$ and $b_{l_{2}}=2$ (the case $b_{l_{1}}<c_{l_{2}}$ is much easier). Then, by condition (3.9), we obtain the approximation (as in (8.24))

$$
\left|\left\langle F_{m, l_{1}}^{n}(j), F_{m, l_{2}}^{n}(j)\right\rangle_{\mathbb{H} \otimes m}\right| \leq C\left(\frac{1}{n} \sum_{j=1}^{n} j \mathrm{r}(j)+\sum_{j=1}^{n-1} \mathrm{r}(n+j)\right) \rightarrow 0,
$$

since $\sum_{j=1}^{\infty} \mathrm{r}(j)<\infty$.

(iv) A straightforward computation shows that

$$
\begin{aligned}
& \left\|F_{m}^{n}(j) \otimes_{p} F_{m}^{n}(j)\right\|_{\mathbb{H} \otimes 2(m-p)}^{2} \\
& \quad=\frac{C_{m, j}}{n^{2}} \sum_{i_{1}, i_{2}, i_{3}, i_{4}=1}^{n-1} r_{n}^{p}\left(\left|i_{1}-i_{2}\right|\right) r_{n}^{p}\left(\left|i_{4}-i_{3}\right|\right) r_{n}^{m-p}\left(\left|i_{1}-i_{4}\right|\right) r_{n}^{m-p}\left(\left|i_{2}-j_{3}\right|\right)
\end{aligned}
$$

for some constant $C_{m, j}$. The latter is smaller than

$$
\begin{aligned}
& \frac{C}{n} \sum_{i, h, l=1}^{n-1}\left|r_{n}^{p}(i)\right|\left|r_{n}^{p}(l)\right|\left|r_{n}^{m-p}(|i-h|)\right|\left|r_{n}^{m-p}(|l-h|)\right| \\
& \quad=\frac{C}{n} \sum_{h=1}^{n-1}\left(\sum_{i=1}^{n-1}\left|r_{n}^{p}(i)\right|\left|r_{n}^{m-p}(|i-h|)\right|\right)^{2} .
\end{aligned}
$$

Now, for any $0<\varepsilon<1$, we obtain by the Hölder inequality

$$
\begin{aligned}
& n^{-1} \sum_{0 \leq h \leq n-1}\left(\sum_{0 \leq i \leq n-1}\left|r_{n}^{p}(i)\right|\left|r_{n}^{m-p}(|i-h|)\right|\right)^{2} \\
& \leq n^{-1} \sum_{0 \leq h \leq[n \varepsilon]}\left(\sum_{0 \leq i \leq n-1}\left|r_{n}^{p}(i)\right|\left|r_{n}^{m-p}(|i-h|)\right|\right)^{2}
\end{aligned}
$$




$$
\begin{aligned}
& +2 n^{-1} \sum_{h=[n \varepsilon]}^{n-1}\left(\sum_{i=0}^{[n \varepsilon / 2]}\left|r_{n}^{p}(i)\right|\left|r_{n}^{m-p}(|i-h|)\right|\right)^{2} \\
& +2 n^{-1} \sum_{h=[n \varepsilon]}^{n-1}\left(\sum_{h=[n \varepsilon / 2]}^{n-1}\left|r_{n}^{p}(i)\right|\left|r_{n}^{m-p}(|i-h|)\right|\right)^{2} \\
& \leq C\left(\varepsilon\left(\sum_{0 \leq i \leq n-1}\left|r_{n}^{m}(i)\right|\right)^{2}+\left(\sum_{0 \leq i \leq n-1}\left|r_{n}^{m}(i)\right|\right)^{2 p / m}\left(\sum_{[n \varepsilon / 2]<h \leq n-1}\left|r_{n}^{m}(h)\right|\right)^{2(m-p) / m}\right) .
\end{aligned}
$$

The latter is smaller (again by (3.9)) than

$$
C\left(\varepsilon\left(\sum_{0 \leq i \leq n-1} \mathrm{r}(i)\right)^{2}+\left(\sum_{0 \leq i \leq n-1} \mathrm{r}(i)\right)^{2 p / m}\left(\sum_{[n \varepsilon / 2]<h \leq n-1} \mathrm{r}(h)\right)^{2(m-p) / m}\right)
$$

that converges to $C \varepsilon\left(\sum_{i=0}^{\infty} r(i)\right)^{2}$ as $n \rightarrow \infty$. Thus, we obtain (iv) by letting $\varepsilon \rightarrow 0$.

Step 2: Clearly, it suffices to consider the case $d=1, p_{l}^{1}=p_{l}$. Set

$$
\begin{aligned}
\sqrt{n}\left(V\left(p_{1}, \ldots, p_{k}\right)_{t}^{n}-\rho_{p_{1}, \ldots, p_{k}}^{(n)} t\right) & =\sum_{m=2}^{\infty} I_{m}\left(\frac{1}{\sqrt{n}} \sum_{i=1}^{[n t]} f_{m}^{n}(i)\right)+\mathrm{O}_{p}\left(n^{-1 / 2}\right) \\
& =: Z_{t}^{n}+\mathrm{O}\left(n^{-1 / 2}\right)
\end{aligned}
$$

(where the approximation holds locally uniformly in $t$ ) and

$$
Z_{t}^{n, N}=\sum_{m=2}^{N} I_{m}\left(\frac{1}{\sqrt{n}} \sum_{i=1}^{[n t]} f_{m}^{n}(i)\right)
$$

In step 1, we have proved that conditions (i)-(iii) of Theorem 2 in [5] are satisfied. Then by (2.3) of Theorem 2 in [5] and the Cauchy-Schwarz inequality, we obtain the approximation

$$
\begin{aligned}
& P\left(\left|Z_{t}^{n, N}-Z_{t_{1}}^{n, N}\right| \geq \lambda,\left|Z_{t_{2}}^{n, N}-Z_{t}^{n, N}\right| \geq \lambda\right) \\
& \leq \frac{E^{1 / 2}\left[\left|Z_{t}^{n, N}-Z_{t_{1}}^{n, N}\right|^{4}\right] E^{1 / 2}\left[\left|Z_{t_{2}}^{n, N}-Z_{t}^{n, N}\right|^{4}\right]}{\lambda^{4}} \\
& \leq C \frac{\beta^{2}\left([n t]-\left[n t_{1}\right]\right)\left(\left[n t_{2}\right]-[n t]\right)}{n^{2} \lambda^{4}} \leq C \frac{\beta^{2}\left(t_{2}-t_{1}\right)^{2}}{\lambda^{4}}
\end{aligned}
$$

for any $t_{1} \leq t \leq t_{2}$ and $\lambda>0$. On the other hand, (8.14) and (8.24) imply that

$$
\lim _{N \rightarrow \infty} E\left[\left|Z_{t}^{n}-Z_{t}^{n, N}\right|^{2}\right]=0
$$


for any $n$ and any $t$. Using this we conclude that

$$
P\left(\left|Z_{t}^{n}-Z_{t_{1}}^{n}\right| \geq \lambda,\left|Z_{t_{2}}^{n}-Z_{t}^{n}\right| \geq \lambda\right) \leq C \frac{\beta^{2}\left(t_{2}-t_{1}\right)^{2}}{\lambda^{4}}
$$

for any $t_{1} \leq t \leq t_{2}$ and $\lambda>0$, from which we deduce the tightness of the sequence $Z_{t}^{n}$ by Theorem 15.6 in [20]. This completes the proof of Theorem 2.

Proof of Theorem 4. We only consider the case $Z_{1}=0$ (if $Z_{1} \neq 0$, we can proceed as in Theorem 3). Also, for the sake of simplicity, we take $d=1, k=1$ and $p_{1}=p$. We use the decomposition from the proof of Theorem 3:

$$
\begin{aligned}
& \sqrt{n}\left(V(Y, p)_{t}^{n}-\mu_{p} \int_{0}^{t}\left|\sigma_{s}\right|^{p} \mathrm{~d} s\right) \\
& =\sqrt{n}\left(\frac{1}{n \tau_{n}^{p}} \sum_{j=1}^{[l t]}\left|\sigma_{(j-1) / l}\right|^{p} \sum_{i \in I_{l}(j)}\left|\Delta_{i}^{n} G\right|^{p}-\mu_{p} l^{-1} \sum_{j=1}^{[l t]}\left|\sigma_{(j-1) / l}\right|^{p}\right) \\
& +\frac{1}{\sqrt{n} \tau_{n}^{p}} \sum_{i=1}^{[n t]}\left(\left|\Delta_{i}^{n} Y\right|^{p}-\left|\sigma_{(i-1) / n} \Delta_{i}^{n} G\right|^{p}\right)+\bar{R}_{t}^{n, l}
\end{aligned}
$$

for any $l \leq n$, with

$$
\begin{aligned}
\bar{R}_{t}^{n, l}= & \frac{1}{\sqrt{n} \tau_{n}^{p}}\left(\sum_{i=1}^{[n t]}\left|\sigma_{(i-1) / n} \Delta_{i}^{n} G\right|^{p}-\sum_{j=1}^{[l t]}\left|\sigma_{(j-1) / l}\right|^{p} \sum_{i \in I_{l}(j)}\left|\Delta_{i}^{n} G\right|^{p}\right) \\
& +\sqrt{n} \mu_{p}\left(l^{-1} \sum_{j=1}^{[l t]}\left|\sigma_{(j-1) / l}\right|^{p}-\int_{0}^{t}\left|\sigma_{s}\right|^{p} \mathrm{~d} s\right) .
\end{aligned}
$$

Observe that under the assumption (CLT) we obtain the weak convergence

$$
\sqrt{n}\left(V(G, p)_{t}^{n}-\mu_{p} t\right) \stackrel{\mathcal{L}}{\rightarrow} \sqrt{\beta} B_{t}
$$

(see Theorem 2). Since $E\left[G_{t}\left(V(G, p)_{t}^{n}-\mu_{p} t\right)\right]=0$ for any $t>0$, because $G$ has a symmetric distribution, we deduce (by Theorem 5 in [3]) that

$$
\left(G_{t}, \sqrt{n}\left(V(G, p)_{t}^{n}-\mu_{p} t\right)\right) \stackrel{\mathcal{L}}{\rightarrow}\left(G_{t}, \sqrt{\beta} B_{t}\right) .
$$

Now, an application of the condition $D^{\prime \prime}$ from Proposition 2 in [1] shows that

$$
\sqrt{n}\left(V(G, p)_{t}^{n}-\mu_{p} t\right) \stackrel{\mathrm{st}}{\longrightarrow} \sqrt{\beta} B_{t} .
$$


By the properties of stable convergence, it follows immediately that

$$
\sqrt{n}\left(\frac{1}{n \tau_{n}^{p}} \sum_{j=1}^{[l t]}\left|\sigma_{(j-1) / l}\right|^{p} \sum_{i \in I_{l}(j)}\left|\Delta_{i}^{n} G\right|^{p}-\mu_{p} l^{-1} \sum_{j=1}^{[l t]}\left|\sigma_{(j-1) / l}\right|^{p}\right) \stackrel{\mathrm{st}}{\longrightarrow} \sqrt{\beta} \sum_{j=1}^{[l t]}\left|\sigma_{(j-1) / l}\right|^{p} \Delta_{j}^{l} B
$$

for any fixed $l$. On the other hand, we have

$$
\sqrt{\beta} \sum_{j=1}^{[l t]}\left|\sigma_{(j-1) / l}\right|^{p} \Delta_{j}^{l} B \stackrel{P}{\longrightarrow} \sqrt{\beta} \int_{0}^{t}\left|\sigma_{s}\right|^{p} \mathrm{~d} B_{s}
$$

as $l \rightarrow \infty$.

Now we need to prove that the other summands in the decomposition (8.25) are negligible. The negligibility of the term $\bar{R}_{t}^{n, l}$ is shown as in the proof of Theorem 7 in [3] but by using condition (4.7) instead of the Hölder continuity of index $\gamma$. So we are left to prove that

$$
\frac{1}{\sqrt{n} \tau_{n}^{p}} \sum_{i=1}^{[n t]}\left(\left|\Delta_{i}^{n} Y\right|^{p}-\left|\sigma_{(i-1) / n} \Delta_{i}^{n} G\right|^{p}\right) \stackrel{P}{\longrightarrow} 0 .
$$

By applying, for $p \geq 1$, the inequality $\left.|| x\right|^{p}-|y|^{p}|\leq p| x-y \mid\left(|x|^{p-1}+|y|^{p-1}\right)$, (8.17) and the Cauchy-Schwarz inequality, and, for $p \leq 1,\left.|| x\right|^{p}-|y|^{p}|\leq| x-\left.y\right|^{p}$ and the Jensen inequality, we have

$$
\left.\frac{1}{\sqrt{n} \tau_{n}^{p}} \sum_{i=1}^{[n t]} E|| \Delta_{i}^{n} Y\right|^{p}-\left|\sigma_{(i-1) / n} \Delta_{i}^{n} G\right|^{p} \mid \leq \frac{1}{\sqrt{n} \tau_{n}^{p \wedge 1}} \sum_{i=1}^{[n t]}\left(E\left|\Delta_{i}^{n} Y-\sigma_{(i-1) / n} \Delta_{i}^{n} G\right|^{2}\right)^{(p \wedge 1) / 2}
$$

Now we use a similar decomposition as presented in the proof of Theorem 3:

$$
\Delta_{i}^{n} Y-\sigma_{(i-1) / n} \Delta_{i}^{n} G=A_{i}^{n}+B_{i}^{n, \varepsilon_{n}^{(1)}}+\sum_{j=1}^{l} C_{i}^{n, \varepsilon_{n}^{(j)}, \varepsilon_{n}^{(j+1)}}
$$

where $A_{i}^{n}, B_{i}^{n, \varepsilon_{n}^{(1)}}$ are defined as above, $0<\varepsilon_{n}^{(1)}<\cdots<\varepsilon_{n}^{(l)}<\varepsilon_{n}^{(l+1)}=\infty$ and

$$
\begin{aligned}
C_{i}^{n, \varepsilon_{n}^{(j)}, \varepsilon_{n}^{(j+1)}}= & \int_{(i-1) / n-\varepsilon_{n}^{(j+1)}}^{(i-1) / n-\varepsilon_{n}^{(j)}}\left(g\left(\frac{i}{n}-s\right)-g\left(\frac{i-1}{n}-s\right)\right) \sigma_{s} W(\mathrm{~d} s) \\
& -\sigma_{(i-1) / n} \int_{(i-1) / n-\varepsilon_{n}^{(j+1)}}^{(i-1) / n-\varepsilon_{n}^{(j)}}\left(g\left(\frac{i}{n}-s\right)-g\left(\frac{i-1}{n}-s\right)\right) W(\mathrm{~d} s) .
\end{aligned}
$$


By the assumption (CLT) and Lemma 2, we obtain the following inequalities (since $\sigma$ is bounded on compact intervals)

$$
\begin{gathered}
\frac{1}{\sqrt{n} \tau_{n}^{p \wedge 1}} \sum_{i=1}^{[n t]}\left(E\left|A_{i}^{n}\right|^{2}\right)^{(p \wedge 1) / 2} \leq C n^{-\gamma(p \wedge 1)+1 / 2}, \\
\frac{1}{\sqrt{n} \tau_{n}^{p \wedge 1}} \sum_{i=1}^{[n t]}\left(E\left|B_{i}^{n, \varepsilon_{n}^{(1)}}\right|^{2}\right)^{(p \wedge 1) / 2} \leq C n^{1 / 2}\left|\varepsilon_{n}^{(1)}\right|^{\gamma(p \wedge 1)}, \\
\frac{1}{\sqrt{n} \tau_{n}^{p \wedge 1}} \sum_{i=1}^{[n t]}\left(E\left|C_{i}^{n, \varepsilon_{n}^{(j)}, \varepsilon_{n}^{(j+1)}}\right|^{2}\right)^{(p \wedge 1) / 2} \leq C n^{1 / 2}\left|\varepsilon_{n}^{(j+1)}\right|^{\gamma(p \wedge 1)} \mid \pi^{n}\left(\left.\left[\varepsilon_{n}^{(j)}, \varepsilon_{n}^{(j+1)}\right)\right|^{(p \wedge 1) / 2},\right. \\
j=1, \ldots, l-1, \\
\frac{1}{\sqrt{n} \tau_{n}^{p \wedge 1}} \sum_{i=1}^{[n t]}\left(E\left|C_{i}^{n, \varepsilon_{n}^{(l)}, \varepsilon_{n}^{(l+1)}}\right|^{2}\right)^{(p \wedge 1) / 2} \leq C n^{1 / 2} \pi^{n}\left(\left(\varepsilon_{n}^{(l)}, \infty\right)\right)^{(p \wedge 1) / 2} .
\end{gathered}
$$

Then we deduce by (CLT) and Lemma 3

$$
\frac{1}{\sqrt{n} \tau_{n}^{p}} \sum_{i=1}^{[n t]}\left|\Delta_{i}^{n} Y-\sigma_{(i-1) / n} \Delta_{i}^{n} G\right|^{p} \stackrel{P}{\longrightarrow} 0 .
$$

This completes the proof of Theorem 4.

\section{Acknowledgements}

Ole E. Barndorff-Nielsen and Mark Podolskij acknowledge financial support from CREATES, funded by the Danish National Research Foundation, and from the Thiele Centre. The work of José Manuel Corcuera is supported by the MEC Grant No. MTM2009-08218.

\section{References}

[1] Aldous, D.J. and Eagleson, G.K. (1978). On mixing and stability of limit theorems. Ann. Probab. 6 325-331. MR0517416

[2] Barndorff-Nielsen, O.E. and Basse-O'Connor, A. (2010). Quasi Ornstein-Uhlenbeck processes. Bernoulli. To appear.

[3] Barndorff-Nielsen, O.E., Corcuera, J.M. and Podolskij, M. (2009). Power variation for Gaussian processes with stationary increments. Stochastic Process. Appl. 119 1845-1865. MR2519347

[4] Barndorff-Nielsen, O.E., Corcuera, J.M. and Podolskij, M. (2009). Multipower variation for Brownian semistationary processes (full version). CREATES research paper 2009-21, Aarhus Univ. Available at http://www.econ.au.dk/research/research-centres/creates/research/research-papers/ research-papers-2009/. 
[5] Barndorff-Nielsen, O.E., Corcuera, J.M., Podolskij, M. and Woerner, J.H.C. (2009). Bipower variation for Gaussian processes with stationary increments. J. Appl. Probab. 46 132-150. MR2508510

[6] Barndorff-Nielsen, O.E., Graversen, S.E., Jacod, J., Podolskij, M. and Shephard, N. (2006). A central limit theorem for realised power and bipower variations of continuous semimartingales. In From Stochastic Calculus to Mathematical Finance. Festschrift in Honour of A.N. Shiryaev (Y. Kabanov, R. Liptser and J. Stoyanov, eds.) 33-68. Heidelberg: Springer. MR2233534

[7] Barndorff-Nielsen, O.E. and Schmiegel, J. (2004). Lévy-based tempo-spatial modelling: With applications to turbulence. Uspekhi Mat. NAUK 59 65-91. MR2068843

[8] Barndorff-Nielsen, O.E. and Schmiegel, J. (2007). Ambit processes: With applications to turbulence and cancer growth. In Stochastic Analysis and Applications: The Abel Symposium 2005 (F.E. Benth, G.D. Nunno, T. Linstrøm, B. Øksendal and T. Zhang, eds.) 93-124. Heidelberg: Springer. MR2397785

[9] Barndorff-Nielsen, O.E. and Schmiegel, J. (2008a). A stochastic differential equation framework for the timewise dynamics of turbulent velocities. Theory Probab. Appl. 52 372-388.

[10] Barndorff-Nielsen, O.E. and Schmiegel, J. (2008b): Time change, volatility and turbulence. In Proceedings of the Workshop on Mathematical Control Theory and Finance (A. Sarychev, A. Shiryaev, M. Guerra and M.D.R. Grossinho, eds.) 29-53. Berlin: Springer. MR2484103

[11] Barndorff-Nielsen, O.E. and Schmiegel, J. (2008c). Time change and universality in turbulence. Research Report 2007-8. Thiele Centre for Applied Mathematics in Natural Science. Unpublished manuscript.

[12] Barndorff-Nielsen, O.E. and Schmiegel, J. (2009). Brownian semistationary processes and volatility/intermittency. In Advanced Financial Modelling. Radon Series Comp. Appl. Math. 8 (H. Albrecher, W. Rungaldier and W. Schachermeyer, eds.) 1-26. Berlin: W. de Gruyter. MR2648456

[13] Barndorff-Nielsen, O.E. and Shephard, N. (2004). Power and bipower variation with stochastic volatility and jumps (with discussion). J. Fin. Econometrics 2 1-48.

[14] Barndorff-Nielsen, O.E. and Shephard, N. (2004). Econometric analysis of realised covariation: High frequency covariance, regression and correlation in financial economics. Econometrica 72 885-925. MR2051439

[15] Barndorff-Nielsen, O.E. and Shephard, N. (2006). Impact of jumps on returns and realised variances: Econometric analysis of time-deformed Lévy processes. J. Econometrics 131 217-252. MR2276000

[16] Barndorff-Nielsen, O.E. and Shephard, N. (2007). Variation, jumps, market frictions and high frequency data in financial econometrics. In Advances in Economics and Econometrics. Theory and Applications. Ninth World Congress (R. Blundell, T. Persson and W.K. Newey, eds.) 328-372. Cambridge Univ. Press.

[17] Barndorff-Nielsen, O.E. and Shephard, N. and Winkel, M. (2006). Limit theorems for multipower variation in the presence of jumps. Stochastic Process. Appl. 116 796-806. MR2218336

[18] Bégyn, A. (2007). Asymptotic expansions and central limit theorem for quadratic variations of Gaussian processes. Bernoulli 13 712-753. MR2348748

[19] Bégyn, A. (2007). Functional limit theorems for generalized quadratic variations of Gaussian processes. Stochastic Process. Appl. 117 1848-1869. MR2437732

[20] Billingsley, P. (1968). Convergence of Probability Measures. New York: Wiley. MR0233396

[21] Cramér, H. and Leadbetter, M.R. (1967). Stationary and Related Stochastic Processes. New York: Wiley.

[22] Guyon, X. and Leon, J. (1989). Convergence en loi des H-variation d'un processus gaussien stationaire. Ann. Inst. H. Poincaré Probab. Statist. 25 265-282. MR1023952

[23] Ho, H.C. and Sun, T.C. (1987). A central limit theorem for non-instantaneous filters of a stationary Gaussian process. J. Multivariate Anal. 22 144-155. MR0890889

[24] Jacod, J. (2008). Asymptotic properties of realized power variations and related functionals of semimartingales. Stochastic Process. Appl. 118 517-559. MR2394762 
[25] Jacod, J. (2008). Statistics and high frequency data. Lecture notes. Department of Statistics, Université Paris VI.

[26] Jacod, J. (2008). Asymptotic properties of realized power variations and related functionals of semimartingales: Multipower variations. Working paper.

[27] Kinnebrock, S. and Podolskij, M. (2008). A note on the central limit theorem for bipower variation of general functions. Stochastic Process. Appl. 118 1056-1070. MR2418258

[28] Nabeya, S. (1952). Absolute moments in 3-dimensional normal distribution. Ann. Inst. Statist. Math. 4 15-30. MR0052072

[29] Nualart, D. (2006). The Malliavin Calculus and Related Topics, 2nd ed. Berlin: Springer. MR2200233

[30] Nualart, D. and Peccati, G. (2005). Central limit theorems for sequences of multiple stochastic integrals. Ann. Probab. 33 177-193. MR2118863

[31] Nualart, D. and Ortiz-Latorre, S. (2008). Central limit theorems for multiple stochastic integrals and Malliavin calculus. Stochastic Process. Appl. 118 614-628. MR2394845

[32] Peccati, G. and Tudor, C.A. (2005). Gaussian limits for vector-valued multiple stochastic integrals. In Seminaire de Probabilites XXXVIII (M. Emery, M. Ledoux and M. Yor, eds.). Lecture Notes in Mathematics 1857 247-262. Berlin: Springer. MR2126978

[33] Woerner, J.H.C. (2006). Power and multipower variation: Inference for high frequency data. In Stochastic Finance (A.N. Shiryaev, M. do Rosário Grossihno, P. Oliviera and M. Esquivel, eds.) 343364. Heidelberg: Springer. MR2230770

[34] Woerner, J.H.C. (2008). Volatility estimates for high frequency data: Market microstructure noise versus fractional Brownian motion models. Unpublished manuscript.

Received June 2009 and revised June 2010 